\title{
Improving the ameliorative effects of berberine and curcumin combination via dextran-coated bilosomes on non-alcohol fatty liver disease in mice
}

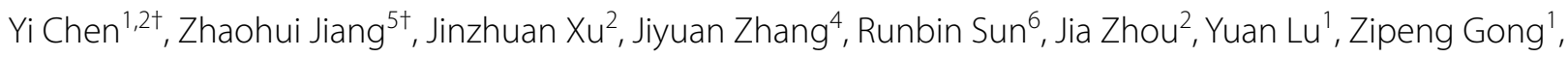
Jing Huang ${ }^{1}$, Xiangchun Shen ${ }^{1,2}$, Qianming Du ${ }^{3,4^{*}}$ and Jianqing Peng ${ }^{1,2^{*}}$ (D)

\begin{abstract}
Background: The combination of berberine (BER) and curcumin (CUR) has been verified with ameliorative effects on non-alcohol fatty liver disease (NAFLD). However, discrepant bioavailability and biodistribution of BER and CUR remained an obstacle to achieve synergistic effects. Multilayer nanovesicles have great potential for the protection and oral delivery of drug combinations. Therein lies bile salts inserted liposomes, named as bilosomes, that possesses long residence time in the gastrointestinal tract (GIT) and permeability across the small intestine. Diethylaminoethyl dextran (DEAE-DEX) is generally used as an outside layer on the nanovesicles to increase the mucinous stability and promote oral absorption. Herein, we developed a DEAE-DEX-coated bilosome with BER and CUR encapsulated (DEAEDEX@LSDBC) for the treatment of NAFLD.
\end{abstract}

Results: DEAE-DEX@LSDBC with 150 nm size exhibited enhanced permeation across mucus and Caco-2 monolayer. In vivo pharmacokinetics study demonstrated that DEAE-DEX@LSDBC profoundly prolonged the circulation time and improved the oral absorption of both BER and CUR. Intriguingly, synchronized biodistribution of BER and CUR and highest biodistribution at liver was achieved by DEAE-DEX@LSDBC, which contributed to the optimal ameliorative effects on NAFLD. It was further verified to be mainly mediated by anti-oxidation and anti-inflammation related pathways

Conclusion: DEAE-DEX coated bilosome displayed promoted oral absorption, prolonged circulation and synchronized biodistribution of BER and CUR, leading to improved ameliorative effects on NAFLD in mice, which provided a promising strategy for oral administration of drug combinations.

Keywords: Berberine, Curcumin, Bilosomes, DEAE-DEX, Oral delivery, NAFLD

*Correspondence: duqianming@njmu.edu.cn; pengjianqing90@126.com ${ }^{\dagger} Y$ i Chen and Zhaohui Jiang contributed equally to this work

1 State Key Laboratory of Functions and Applications of Medicinal Plants, Guizhou Medical University, Guiyang 550014, China

${ }^{3}$ General Clinical Research Center, Nanjing First Hospital, Nanjing Medical University, Nanjing 210006, China

Full list of author information is available at the end of the article

\section{Background}

Non-alcohol fatty liver disease (NAFLD) is characterized hepatic steatosis in the absence of heavy alcohol consumption that caused by the overuse of hepatotoxic medication, disorders affecting the liver such as hepatitis $C$ virus infection, Wilson's disease and starvation [1, 2 ]. It had affected approximately $25 \%$ of the global population and high prevalence was observed in patients

c) The Author(s) 2021. This article is licensed under a Creative Commons Attribution 4.0 International License, which permits use, sharing, adaptation, distribution and reproduction in any medium or format, as long as you give appropriate credit to the original author(s) and the source, provide a link to the Creative Commons licence, and indicate if changes were made. The images or other third party material in this article are included in the article's Creative Commons licence, unless indicated otherwise in a credit line to the material. If material is not included in the article's Creative Commons licence and your intended use is not permitted by statutory regulation or exceeds the permitted use, you will need to obtain permission directly from the copyright holder. To view a copy of this licence, visit http://creativecommons.org/licenses/by/4.0/. The Creative Commons Public Domain Dedication waiver (http://creativecommons.org/publicdomain/zero/1.0/) applies to the data made available in this article, unless otherwise stated in a credit line to the data. 
with metabolic derangements [3]. One third of NAFLD patients might develop into nonalcoholic steatohepatitis (NASH), which associates with steatosis, hepatocyte swelling and lobular inflammation, resulting in liver fibrosis and cirrhosis, even hepatic failure and hepatocellular carcinoma (HCC) [4]. Given that hepatic failure is irreversible, amelioration of NAFLD on time to prevent the liver fibrosis and reduce the occurrence of hepatitisrelated and extrahepatic diseases can effectively diminish the mortality induced by hepatic failure [5]. However, due to the not fully understood pathogenesis, there is a lack of ideal drugs for the treatment of NAFLD. So far, the multiple-hit hypothesis for the pathogenesis of NAFLD has been widely accepted. It holds that the development and progression of NAFLD resulted from the multiple damages, including insulin resistance, adipose tissue dysfunction, intestinal flora disorders, oxidative stress damage, genetic and epigenetic factors [6,7]. Since the complexity of NAFLD as a metabolic disease, it is necessary and highly effective to apply multitarget therapies on it.

In recent years, many herbal extracts have been proved with great potential for the treatment of NAFLD, including flavonoids, alkaloids, polysaccharides, volatile oils, quinones, terpenes, coumarins, lignans, saponins, cardiac glycosides, phenolic acids, and amino acids [8]. These extracts alleviated lipid infiltration at liver and improved related anthropometric, hemodynamic and biochemical parameters via immunomodulatory, antioxidant, antiinflammatory, and anti-fibrosis properties with low toxicity $[8,9]$. Among them, berberine (BER) and curcumin (CUR) exhibited promising ameliorative therapeutic effects on NAFLD.

$\mathrm{BER}$ as an isoquinoline alkaloid extracted from Coptis chinensis has been used for diarrhea, inflammatory diseases, and metabolic disorders including obesity and diabetes [10]. Although the mechanisms of BER in the treatment of NAFLD are unclear, a variety of potential targeting pathophysiological processes has been proposed, including increase of hepatic insulin sensitivity [11], reduction of serum cholesterol by stabilizing mRNA of low density lipoprotein receptor (LDLR) [12], enhancement of mitochondrial function to reduce oxidative stress [13] and regulation on adenosine monophosphate activated protein kinase (AMPK) pathway [14]. Similar but not identical, CUR as a natural polyphenol isolated from Curcuma longa has diverse effects of anti-oxidation, anti-inflammation, anti-mutagenesis, anti-fibrosis and reduction on the liver steatosis $[15,16]$. The combination of BER and CUR exerted superior therapeutic effect on NAFLD in rats in contrast to the single drug or lovastatin (a traditional cholesterol-lowering agent) by regulating lipid metabolism, reducing oxidative stress and ameliorating liver inflammation [17]. However, the combination of BER and CUR remains unsatisfied due to the potential toxicity induced by high dosage, especially BER-induced gastrointestinal tract (GIT) damages at higher than $50 \mathrm{mg} / \mathrm{kg}$ in rats via oral administration [18]. The low oral absorption, rapid metabolism and enhanced excretion of BER and CUR are the main reasons for the poor oral bioavailability, which limited the pharmacological actions of the combination [19-21]. Therefore, a co-delivery nanoplatform for BER and CUR combination is highly in need for promoting the ameliorative effects on NAFLD with low oral dosage via improved oral absorption, prolonged circulation and synchronized biodistribution to liver.

In terms of nanoplatforms for oral co-delivery of drugs, liposomes were considered as efficient vehicles for both hydrophilic and hydrophobic via interactions between liposomes and intestinal cells leading to membrane fusion and endocytosis [22, 23]. Although cationic liposome was verified with higher cellular uptake and transportation across intestinal cells integrally in comparison with anionic or neutral liposomes, it failed to maintain the integrity of the majority of liposomes in GIT before absorption resulting in drug leakage $[24,25]$. Therefore, liposomal carriers with better stability and longer residence time in the GIT have been researched. Bile salts are generally used to reinforce liposomes against complex GIT environment, which named as bilosomes. The insertion of bile salts could effectively stabilize the lipid bilayer against the destruction mainly from endogenous bile acids, thus prolonged the residence time of bilosomes in the GIT [26]. Meanwhile, the presence of bile salts enhanced absorption of bilosomes across the intestinal membranes via both trans-enterocytic internalization and paracellular permeation [27]. There is good correlation between particles size of liposomal preparations and the permeation ability. A particle size less than $400 \mathrm{~nm}$ is preferred regardless of the trans-enterocytic internalization and paracellular permeation [28]. Moreover, coating a cover on the surface of liposomes is a good strategy to further prevent the destruction of liposomes in the GIT, thereby maintaining the smaller size and enhancing the stability. Diethylaminoethyl dextran (DEAE-DEX), a cationic polymer, had been used as the cover on the surface of nanoparticles to improve mucinous stability in the GIT fluids, inhibit opsonization in the circulation and promote favorable uptake by hepatocytes via anionic moiety of the asialoglycoprotein receptor (ASGPR) $[29,30]$. Besides, cationic polymer-modified liposomes have been reported to prolong residence time via adhesion to intestinal wall mucus as well [31]. Herein, we speculated that the DEAE-DEX coated bilosomes have great potential to protect the bilosomes in the GIT resulting in promoted oral absorption of drugs. 
Based on these considerations, we developed DEAEDEX coated bilosomes that composed of a type of bile salts-sodium deoxycholate (SDC), soybean lecithin (SPC), cholesterol (CHOL), octadecylamine (ODA) with BER and CUR encapsulated (DEAE-DEX@LSDBC) for the treatment of NAFLD via oral delivery (Scheme 1). It realized elevated trans-mucus potential, enhanced absorption, prolonged blood circulation, synchronized biodistribution and improved therapeutic efficacy on NAFLD compared with free BER and CUR combination. Thus, this work proposed an effective multilayer nanovesicles for drug combinations to treat NAFLD via oral administration.

\section{Results and discussion}

\section{Preparation and optimization of DEAE-DEX@LSDBC}

As a hydrophilic/hydrophobic drug combination, the BER and CUR were encapsulated into bilosomes by a sequential drug loading process to receive $\mathrm{LSDBC}$, in which the common thin-film dispersion was used for CUR followed by a remote loading method for BER. DEAE-DEX was added dropwise into LSDBC under mild stirring to prepare DEAE-DEX@LSDBC. Comparing with LSDBC, a fluffy periphery layer was clearly observed on the outside of DEAE-DEX@LSDBC by TEM. After lyophilization, a yellow spongy solid was obtained (Fig. 1).

Based on the previous developed liposomal preparations for drug combination, several key factors in the formulation and preparation of LSDBC that closely related to the drug loading and characteristics of the bilosomes were optimized. Both the external phase $\mathrm{pH}$ and incubation time showed no effect on the encapsulation of CUR, while BER exhibited highest LR and LC as the external phase at $\mathrm{pH} 7$ and incubation for $30 \mathrm{~min}$ (Additional file 1: Figure S1). In terms of the SPC:CHOL weight ratio, the encapsulation of CUR was reduced as the ratio of $\mathrm{CHOL}$ increased, indicating the competitive relation between CUR and CHOL at the same position in the lipid layer. BER received the highest $L C$ and $L R$ at the SPC:CHOL weight ratio of $10: 1$, which might be attributed to the optimum flexibility of lipid layer in favor of BER encapsulation in the hydrophilic core. The highest LC of BER was received at a SPC:BER weight ratio of 40:7 reaching the maximum loading capacity, higher concentration of BER reduced both LR and LC on the contrary (Additional file 1: Figure S2).

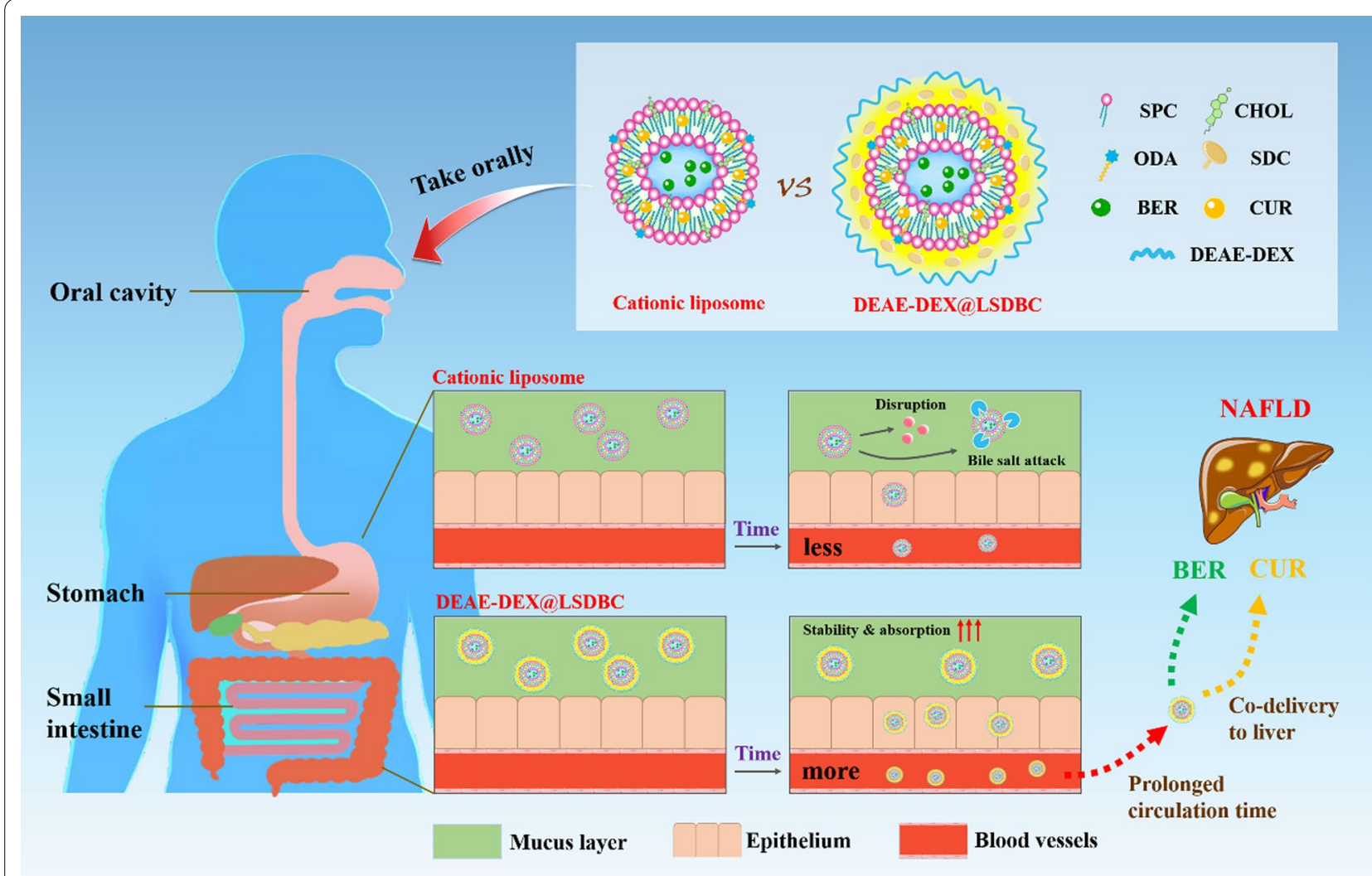

Scheme 1 The superiority of DEAE-DEX@LSDBC over cationic liposomes indicated by promoted oral absorption, prolonged circulation and synchronized biodistribution of BER and CUR especially at liver leading to enhanced ameliorative effects on NAFLD 


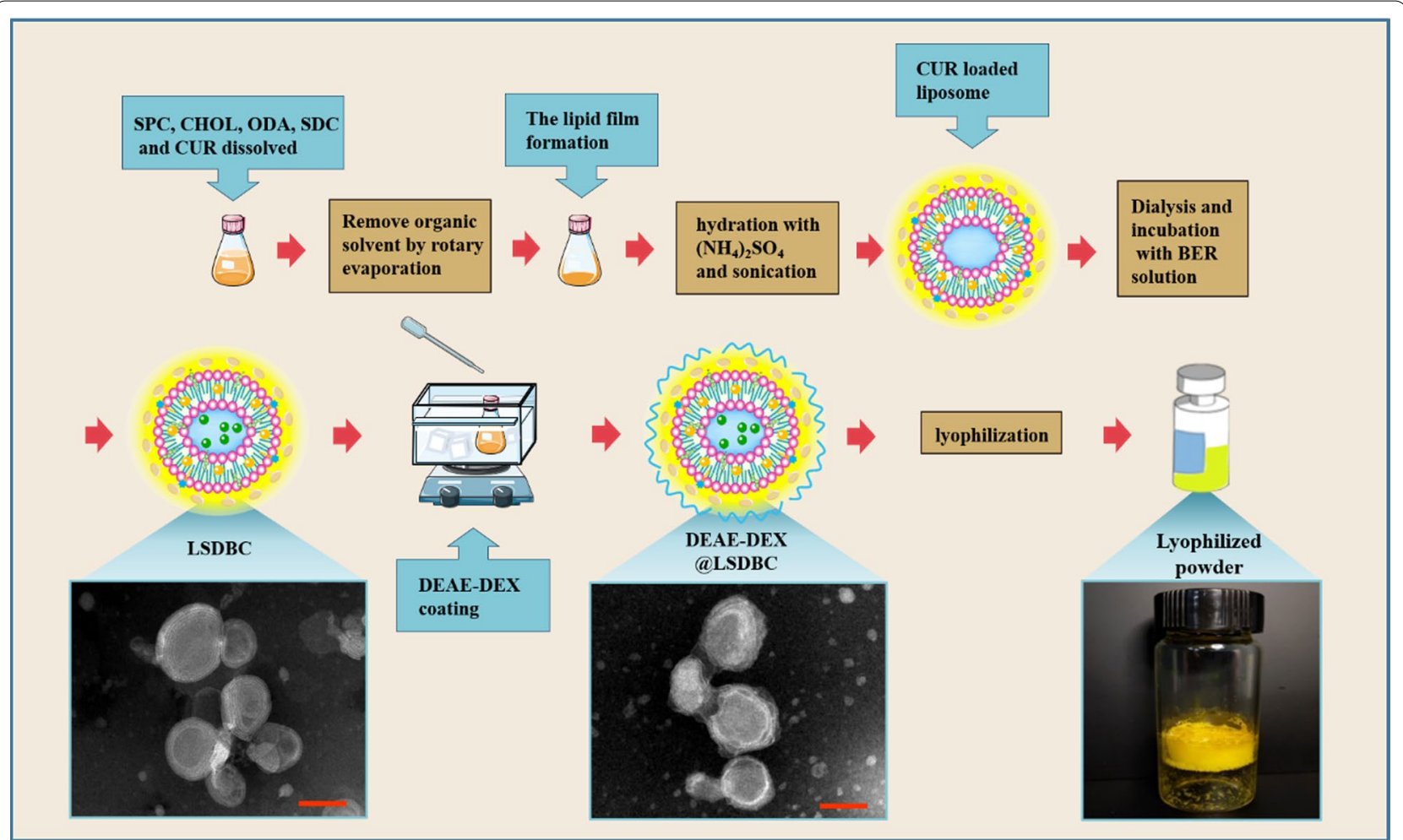

Fig. 1 Schematic illustration of the preparation procedure of DEAE-DEX@LSDBC. Typical patterns of preparations are included, such as the TEM images of LSDBC and DEAE-DEX@LSDBC (scale bar $100 \mathrm{~nm}$ ) and the lyophilized morphology of DEAE-DEX@LSDBC

To steadily absorb DEAE-DEX on the periphery of LSDBC, optimization on the ratio of ODA to SDC is necessary. The decreased molar ratio of ODA:SDC showed little interference on the diameter and PDI of LSDBC, whereas, a charge reversal was detected at 1:3 and no more rise on the negative charge as the ratio decrease (Fig. 2A, C). Thus, the molar ratio of ODA:SDC at 1:3 was used in the preparation of LSDBC to receive negatively charged bilosomes. The content of DEAE-DEX was optimized in terms of diameter, PDI, zeta potential of DEAE-DEX@LSDBC (Fig. 2B, D). Hardly any size variation was observed below $0.39 \mathrm{mg} / \mathrm{mL}$ of DEAE-DEX. A charge reversal was detected at $0.39 \mathrm{mg} / \mathrm{mL}$ of DEAEDEX approaching to electrical neutrality. Meanwhile, the highest permeability across mucus layer of DEAEDEX@LSDBC was detected at the same concentration of DEAE-DEX regardless of the drug (Additional file 1: Figure S3). It confirmed the contribution of hydrophilicity and neutral surface of bilosomes to its mucinous stability. Therefore, the DEAE-DEX at $0.39 \mathrm{mg} / \mathrm{mL}$ was adopted to prepare DEAE-DEX@LSDBC.

\section{Characterization of BER and CUR encapsulated bilosomes}

The TEM images showed nearly spherical particles of LSDBC and DEAE-DEX@LSDBC with similar size
(Fig. 1). In consistent with the images, the hydrodynamic diameters of LBC, LSDBC and DEAE-DEX@LSDBC measured by dynamic light scattering were approximately $150 \mathrm{~nm}$ with a PDI less than 0.3 (Fig. 3A). The charge reversal responding to the SDC insertion and DEAE-DEX coating were detected, respectively (Fig. 3D). Moreover, the UV absorption spectra showed that the characteristic peaks of CUR ( $425 \mathrm{~nm}$ ) and BER (352 and $268 \mathrm{~nm}$ ) were overlapped in drug-encapsulated bilosomes (Additional file 1: Figure S4) indicating the successful encapsulation of BER and CUR. Compared with LBC, the LR and LC of both BER and CUR reduced after the SDC insertion (Fig. 3B, E). It was hypothesized that the insertion of SDC into the lipid bilayer occupied the position of CUR and the negative charge of SDC impeded the encapsulation of BER into internal aqueous phase of bilosomes. Whereas, no more reduction on the LC and LR was detected after DEAE-DEX coating. The final weight ratio of BER and CUR was about 3-1 in all liposomal preparations.

In vitro drug release study was carried out in the SGF and SIF medium under sink condition for both BER and CUR (Fig. 3C, F; Additional file 1: Figure S8). BER showed burst release in the SGF ( $\mathrm{pH}$ 1.2) medium at initial $1 \mathrm{~h}$ and the release rate slowdown in the SIF $(\mathrm{pH}$ 6.8) medium reaching $50 \%$ cumulative release at $24 \mathrm{~h}$. In 

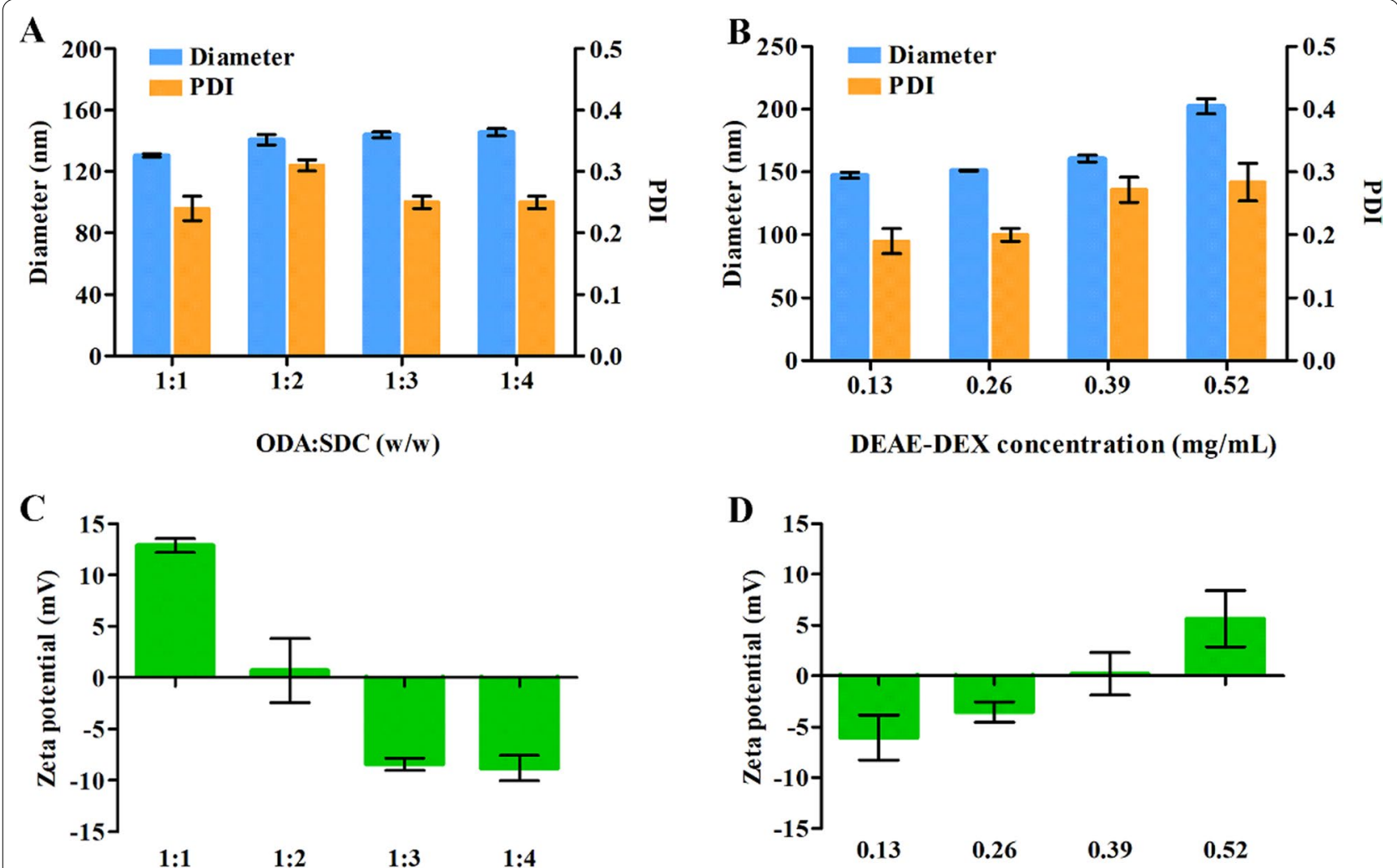

ODA:SDC (w/w)

DEAE-DEX concentration $(\mathrm{mg} / \mathrm{mL})$

Fig. 2 Optimization on the weight ratio of ODA to SDC in terms of $\mathbf{A}$ diameter, PDI and $\mathbf{C}$ zeta potential of LSDBC. The concentrations of DEAE-DEX in DEAE-DEX@LSDBC were optimized in terms of $\mathbf{B}$ diameter, PDI and $\mathbf{D}$ zeta potential. Each bar represents the mean $\pm \mathrm{SD}(n=3)$
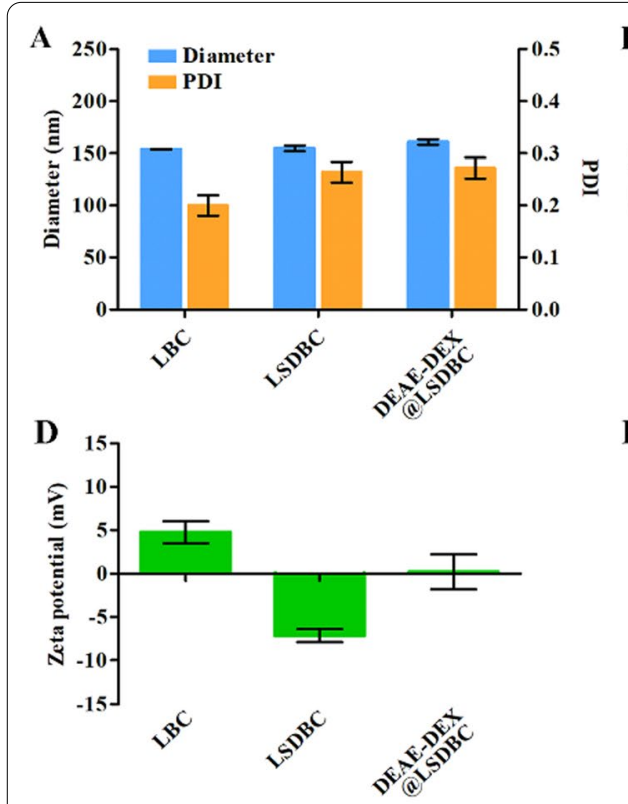
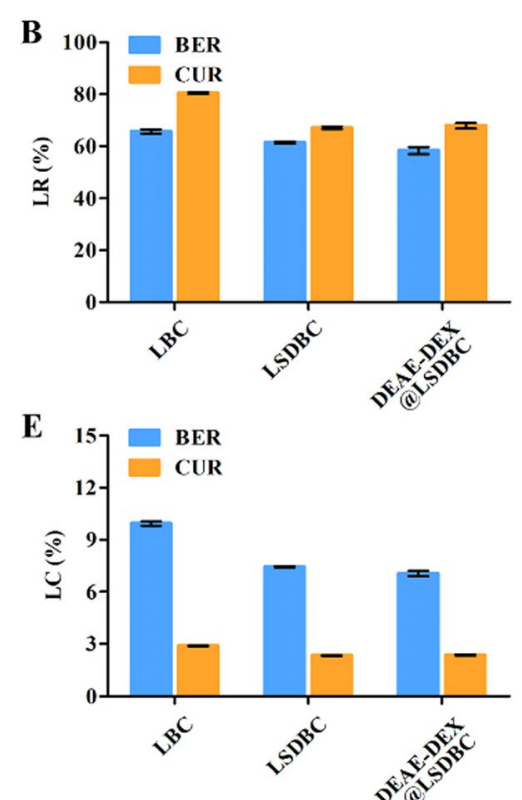
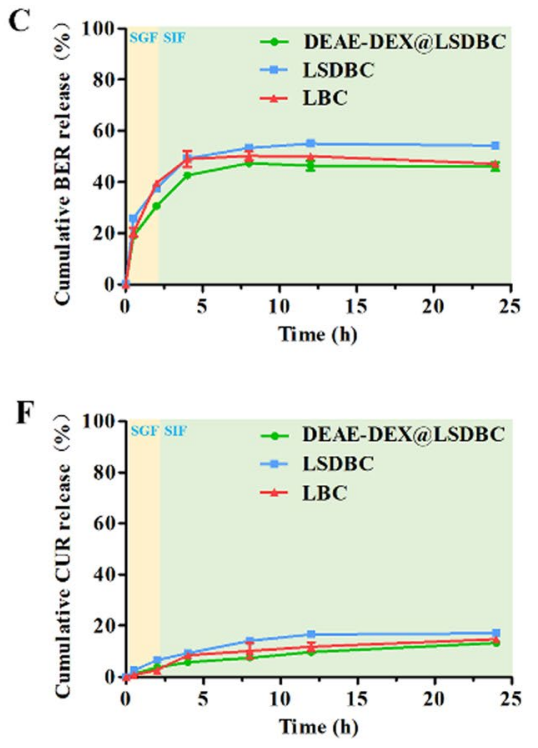

Fig. 3 Characterization of the liposomal preparations. A Diameter, PDI and $\mathbf{D}$ Zeta potential of the preparations. $\mathbf{B} L R$ and $\mathbf{E} L C$ of the preparations. $\mathbf{C}$ BER and $\mathbf{F}$ CUR releases from DEAE-DEX@LSDBC in SFG $(0-2 \mathrm{~h})$ and SIF $(2-24 \mathrm{~h})$ in vitro. Each bar represents the mean \pm SD $(n=3)$ 
comparison with BER, CUR showed lower drug release rate and less than $20 \%$ cumulative release at $24 \mathrm{~h}$. It was speculated that the low $\mathrm{pH}$ of SIF reduced the gel-like precipitate formed between $\mathrm{BER}$ and $\mathrm{SO}_{4}{ }^{2-}$ in the inner aqueous phase of bilosomes and promoted the release of BER. While, CUR interacted with lipid bilayer via mainly hydrophobic interactions was hardly affected by the media. Noteworthy, DEAE-DEX@LSDBC exhibited slight retardation on the drug release compared with LSDBC indicating the weak interactions between DEAEDEX and LSDBC, which guaranteed the shell separation of DEAE-DEX to promote the interactions between LSDBC and intestine.

\section{Permeation across mucus layer and Caco-2 cell monolayer} After oral administration, the intestinal mucus and enterocytes limited the permeation and transportation of drugs into the systemic circulation. To evaluate the adsorption promotion effects of liposomal preparations on the drug combination, in vitro mucus layer (Fig. 4AC) and Caco-2 cell monolayer (Fig. 4D-F) was established to mimic the intestinal absorption barriers. At $0.5,1$ and $2 \mathrm{~h}$, the trans-mucus content of BER and
CUR were detected. The permeability of BER was obviously promoted with time and DEAE-DEX@LSDBC showed optimal permeability (Fig. 4B). However, the ratio of trans-mucus of CUR exhibited indistinctive time-dependent manner in all the groups and weaker advantages of DEAE-DEX@LSDBC on permeation enhancement (Fig. 4C). We speculated that the neutral surface of DEAE-DEX@LSDBC improved the mucinous stability leading to increased permeability of drugs across mucus layer, especially for BER encapsulated in the inner aqueous phase. The inferior promotion on the transmucus percentage of CUR might attribute to the weak protection effects of DEAE-DEX on the lipid bilayer where CUR was inserted.

To evaluate the permeation across enterocytes, the $\mathrm{P}_{\text {app }}$ values of BER and CUR across Caco-2 cell monolayer were calculated. Above all, all the preparations were verified with less than $40 \%$ inhibition on Caco- 2 cells for $24 \mathrm{~h}$ at the concentration of BER $90 \mu \mathrm{g} / \mathrm{ml}$ and CUR $30 \mu \mathrm{g} /$ $\mathrm{ml}$ (Additional file 1: Figure S5), which was considered with low cytotoxicity for only $2 \mathrm{~h}$ incubation in the following experiment. The $\mathrm{P}_{\text {app }}$ value of BER in LSDBC and DEAE-DEX@LSDBC was approximately four-fold of that

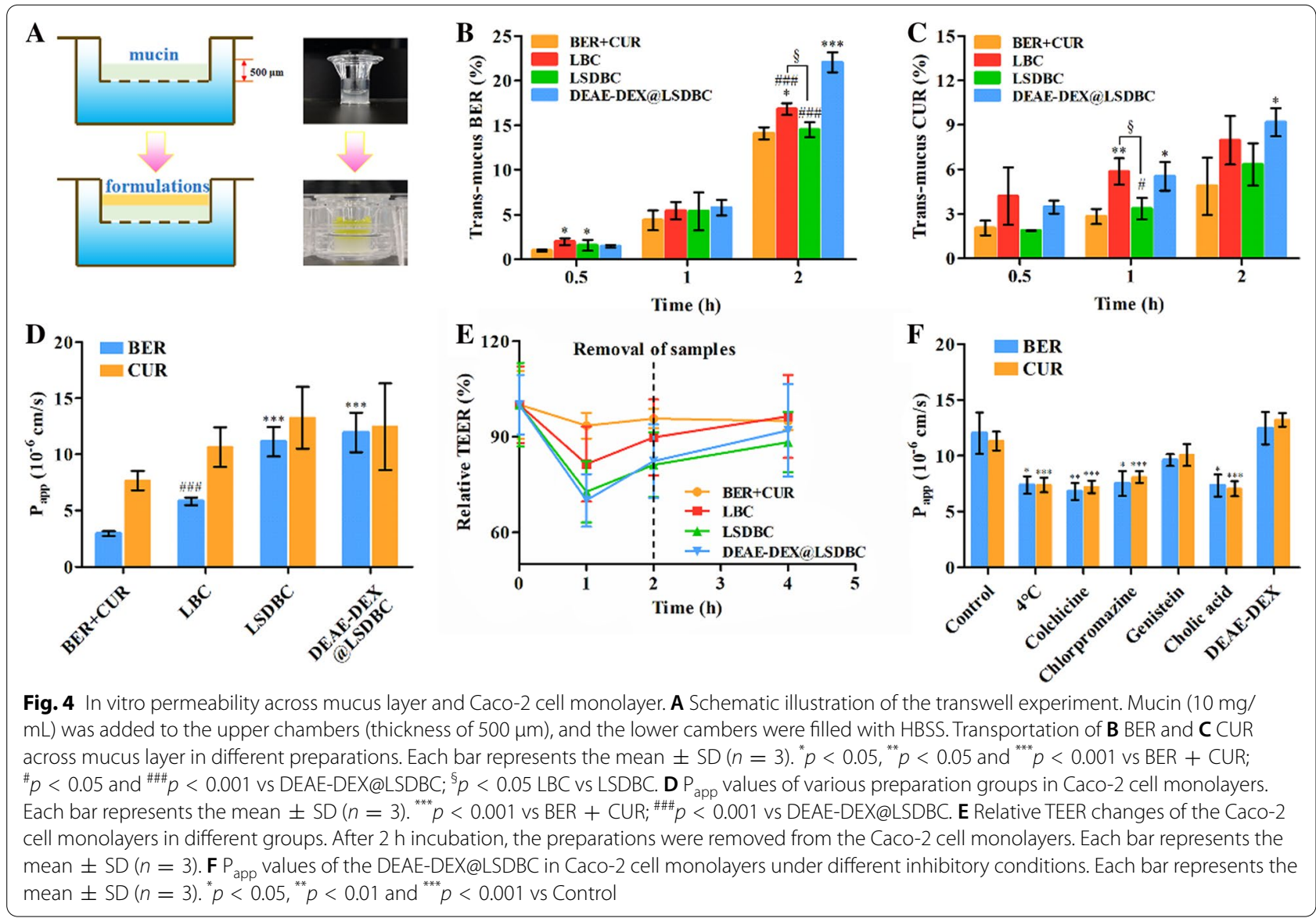


of BER + CUR, while the $\mathrm{P}_{\text {app }}$ value of CUR was only 1.7 -fold increased. Importantly, the obvious difference between the permeability of BER and CUR was coordinated by LSDBC and DEAE-DEX@LSDBC (Fig. 4D) which provided solid foundation for BER and CUR to maintain the initial ratio of drugs after absorption.

The TEER study was generally used to predict the contribution of paracellular permeation in the oral absorption. As shown in Fig. 4E, all the liposomal preparations on the apical side of monolayers induced immediate reduction in TEER in contrast with no reduction at all for BER + CUR. The reduction in TEER was more significant for bilosomes, indicating prominent enhancement of bilosomes on paracellular permeability, which might contribute to the effect of SDC on the tight junctions. Moreover, the gradually increased TEER after removal of preparations indicated recovery of the tight junctions. Thus, it is suggested that bilosomes could transiently and reversibly open the tight junctions in Caco-2 cell monolayers, which is the main mechanism for paracellular permeation. Furthermore, the effect of transenterocytic internalization on the permeation promotion of DEAE-DEX@LSDBC was investigated on the Caco-2 cell monolayer (Fig. 4F). Low temperature, colchicine, chlorpromazine and cholic acid significantly suppressed the $\mathrm{P}_{\text {app }}$ value of both $\mathrm{BER}$ and CUR, indicating the trans-enterocytic internalization of DEAE-DEX@LSDBC mainly via micropinocytosis, clathrin and bile acid transporter-mediated transcellular pathways in an energydependent process. In short, the promotion of both paracellular permeation and trans-enterocytic internalization contributed to enhance permeation across Caco-2 cell monolayer by bilosomes.

\section{Pharmacokinetics study on BER and CUR}

Oral absorption promotion of BER and CUR with the help of liposomal preparations were verified by the altered plasma concentration-time curves of drugs in the male Kunming mice (Fig. 5). Above all, DEAEDEX@LSDBC exhibited the best absorption promotion effect on both BER and CUR. LSDBC and DEAE-DEX@ LSDBC obviously improved the maximum plasma concentration $\left(\mathrm{C}_{\max }\right)$ and area under the curve (AUC) of BER or CUR compared with BER + CUR and LBC groups. As a hydrophilic drug, free BER showed extremely flat plasma concentration-time curve. We speculated that the instability of the LBC in GIT fluids might induce disruption on the structure of carrier and leakage of drugs, especially BER loaded in the internal aqueous phase. On the other hand, given that free CUR possessed superior oral bioavailability than free BER, the difference on plasma concentration-time curves narrowed between BER + CUR and LBC groups.

Moreover, the major pharmacokinetics parameters of BER and CUR were acquired by the compartmental and non-compartmental model fitting of plasma concentration-curve profiles (Additional file 1: Tables S1; S2). The half-life $\left(\mathrm{T}_{1 / 2}\right)$ and mean retention time (MRT) of both BER and CUR manifested an excellently prolonged circulation time by the liposomal preparations. DEAE-DEX@ LSDBC improved the $\mathrm{AUC}_{0-\infty}$ of BER and CUR for more than 10 times of the BER + CUR. The oral bioavailability of BER was 34-fold increased by DEAE-DEX@LSDBC, while only 13-fold increase was observed in CUR compared with BER + CUR. It was reasonable to attribute the superior absorption and longer circulation time of $\mathrm{BER}$ to the drug loading position in the inner water phase
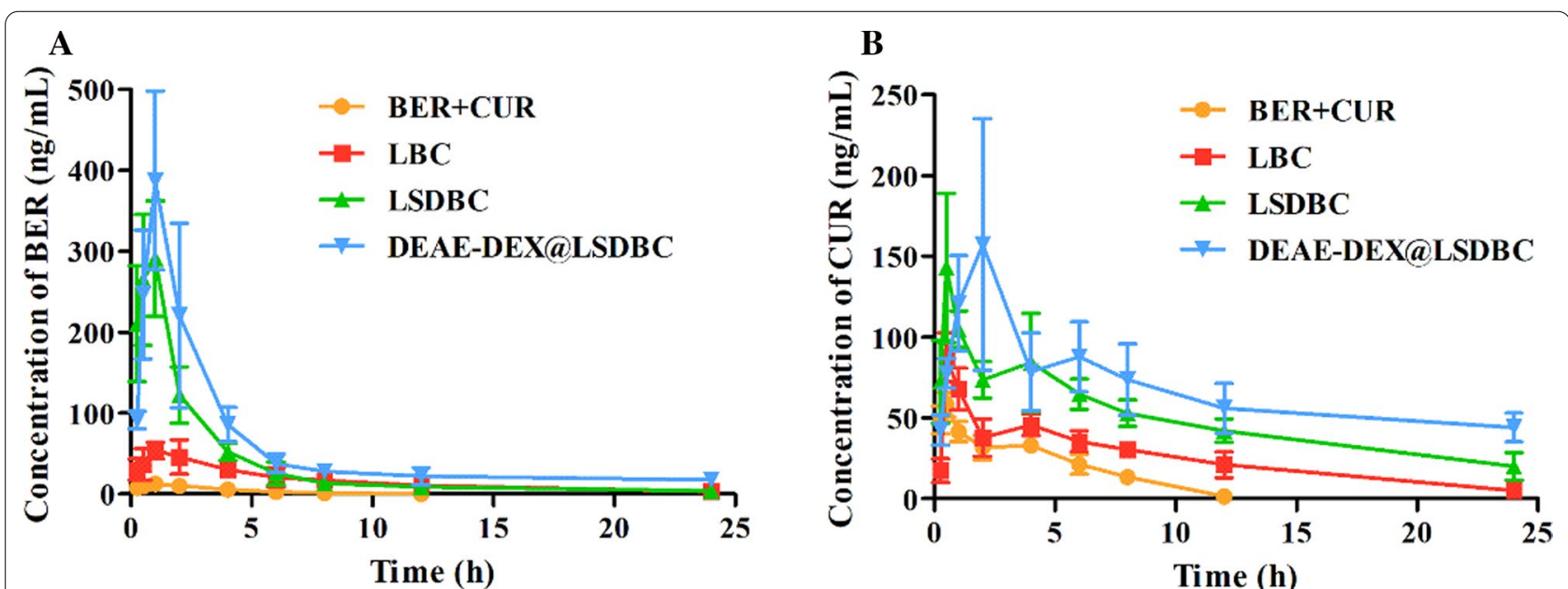

Fig. 5 Plasma concentration-time profiles of $\mathbf{A} B E R$ and $\mathbf{B}$ CUR following the oral administration of various preparations at a dose of $45 \mathrm{mg} / \mathrm{kg}$ BER and $15 \mathrm{mg} / \mathrm{kg}$ CUR in the male Kunming mice. Each bar represents the mean $\pm \mathrm{SD}(n=5)$ 
of bilosomes. The optimal oral absorption promotion and the longest systemic circulation for both BER and CUR was realized by DEAE-DEX@LSDBC. We speculated that the promoted stability of DEAE-DEX@LSDBC in GIT fluids and enhanced permeation across mucus layer and enterocytes contributed to the remarkably promoted oral absorption of BER and CUR.

\section{In vivo biodistribution of $B E R$ and CUR}

The biodistribution of BER and CUR from liposomal preparations were further investigated in the male Kunming mice. After oral administration, the major organs were harvested at 1, 6 and $24 \mathrm{~h}$ for the drug concentration detection. The concentration of both drugs reached peak at $1 \mathrm{~h}$ in each organ and favored the accumulation at liver (Fig. 6A, B). Both drugs showed reduced accumulation in each organ over time in all groups (Fig. 6C-F). It is noteworthy that DEAE-DEX@LSDBC and LSDBC obviously increased the accumulation of BER and CUR at liver compared with BER + CUR and LBC, especially for BER. Intriguingly, the content of BER is 3.2-fold of CUR at liver in DEAE-DEX@LSDBC group at $1 \mathrm{~h}$, which is a little higher than the initial ratio of encapsulated BER and CUR that might be attributed to the better promotion effects on BER loading in the inner phase of bilosomes. DEAE-DEX@LSDBC exhibited the optimal liver targeting capability in terms of the BER and CUR, although no significant difference was observed between

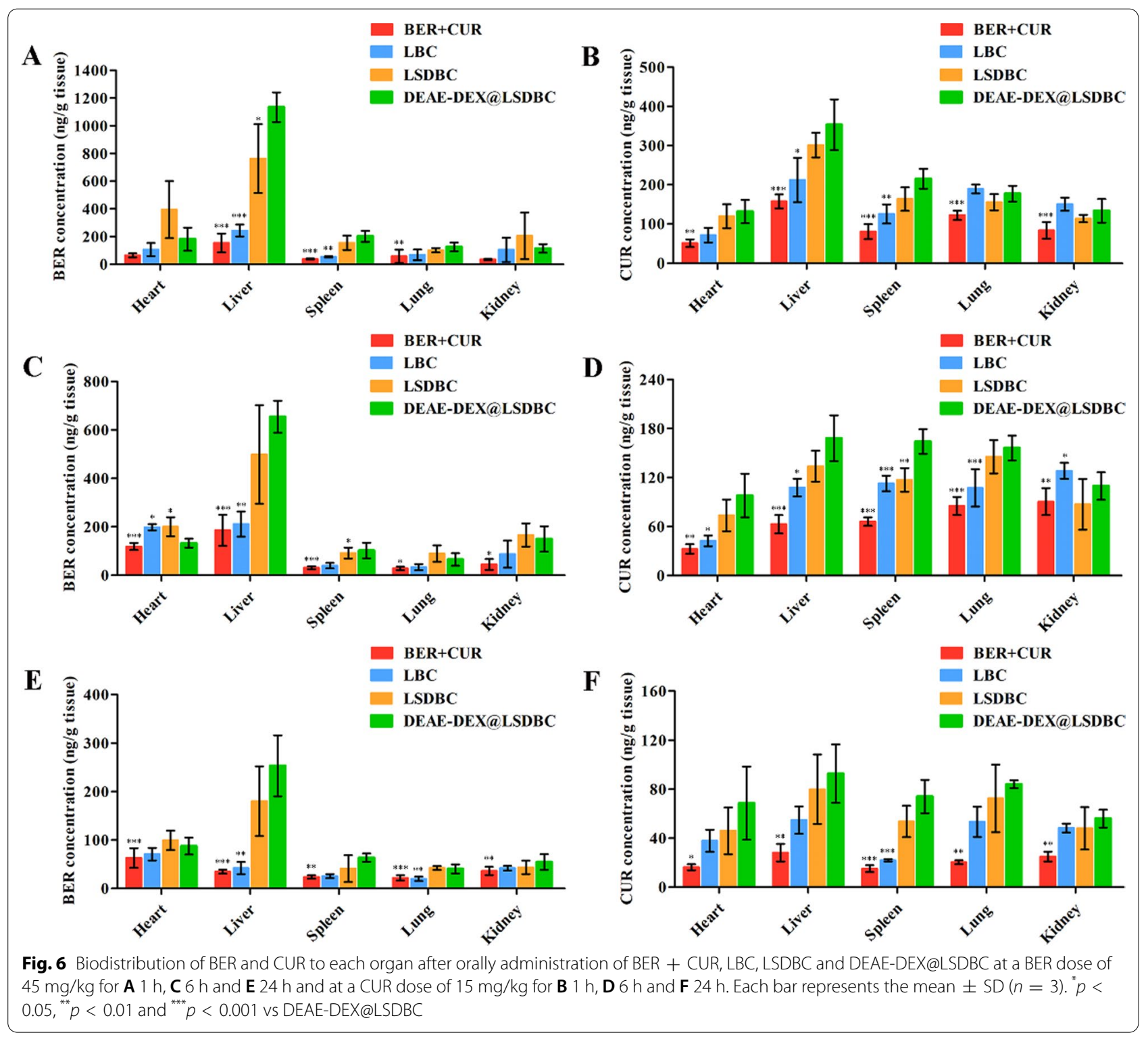


DEAE-DEX@LSDBC and LSDBC after 6 h. In contrast, the content of BER and CUR were comparative in liver at $1 \mathrm{~h}$ between BER + CUR and LBC groups. It is suggested that the DEAE-DEX@LSDBC effectively maintained integrity during oral delivery and achieved highest biodistribution of BER and CUR at liver via enhancing the oral absorption and promoting liver accumulation.

Given that synchronized biodistribution of BER and CUR at targeting organs is of great importance to realize the synergistic effects of the combination, the tissue to plasma $(\mathrm{T} / \mathrm{P})$ ratio of drug was further calculated to evaluate the synchronism biodistribution in vivo (Additional file 1: Figure S6). All the liposomal preparations showed comparative T/P ratios of BER and CUR, especially at $1 \mathrm{~h}$, which would benefit the amelioration effects of drug combination on NAFLD. However, the difference between the T/P ratio of BER and CUR in the BER + CUR group was magnified over time. It is reasonable to suggest that the different in vivo destiny between BER and CUR was efficiently coordinated by the liposomal carriers regardless of the SDC insertion and DEAEDEX coating.

\section{Ameliorative effects of the preparations on NAFLD in mice} Above all, the anti-lipid accumulation effects of free BER, CUR, BER + CUR and bilosomes of the combination were detected on the free fatty acids (FFA) cultured LO2 cell model. All the preparations at an equivalent concentration of BER $3 \mu \mathrm{g} / \mathrm{ml}$ and CUR $1 \mu \mathrm{g} / \mathrm{ml}$ that was used in the anti-lipid accumulation study exhibited less than $10 \%$ inhibition effect on LO2 cells (Additional file 1: Figure S5). The Oil-red-O staining was used to visualize antilipid accumulation effects (Additional file 1: Figure S7). It was shown that BER + CUR, LBC, LSDBC and DEAEDEX@LSDBC treatments caused remarkable reduction on the fat accumulation in the cytosol of $\mathrm{LO} 2$ cells, while BER and CUR alone possessed inferior anti-lipid accumulation effect in comparison with the BER and CUR combination.

NAFLD mice model was established with high fat \& sucrose diet and preventively treated by BER and CUR combination following the regimen (Fig. 7A). We monitored the body weight every week and measured the fat weight at the end of the feeding study (Fig. 7B, C). Since the obesity is closely associated with NAFLD, gradually increased body weight of Model group might be one of the indicators for successful model establishment. It was further confirmed by the fat weight detected at the end of experiment. LSDBC and DEAE-DEX@LSDBC showed more flat body weight-time curve, which was in consistent with the lower fat weight. The optimal protective effect of DEAE-DEX@LSDBC on inhibiting fat accumulation was clearly verified by the BFR (Fig. 7C). In addition to fat accumulation at liver, the insulin resistance was recognized as one of the first hit of NAFLD genesis. Therefore, the FSG level after treatments was determined to evaluate the effect of BER and CUR combination on the glucose metabolism. All the treatment groups showed decreased FSG at the end of study although it was still higher than normal group. DEAE-DEX@LSDBC and LSDBC exerted better hypoglycemic effect in contrast to BER + CUR and LBC groups (Fig. 7D).

Histopathology analysis on the liver has been used as the golden standard for identification of fatty liver. HE (Fig. 7E) and Oil Red O staining (Fig. 7F) verified the widespread lipid vacuoles and inflammatory foci on the liver of mice in the Model group. At the oral dosage of BER $45 \mathrm{mg} / \mathrm{kg}$ and CUR $15 \mathrm{mg} / \mathrm{kg}$, DEAE-DEX@LSDBC and LSDBC exhibited significantly reduced lipid vacuoles and lesion of inflammation compared with model and BER + CUR group. LBC showed slight reduced lipid vacuoles, indicating inferior ameliorative effect on the fat accumulation than the bilosomes. DEAE-DEX@ LSDBC treatment received the most conspicuous protective effect via preventing lipid droplet accumulation and ameliorating inflammatory injury. Moreover, the serum lipids including TC, TG, HDL-c and LDL-c were measured (Fig. 8A). The drug combination treatments significantly decreased the level of TC, TG, LDL-c and slightly improved the HDL-c level, indicating that BER and CUR combination effectively ameliorated the serum lipid accumulation, especially for DEAE-DEX@LSDBC group. Meanwhile, the degree of liver injury was generally evaluated by the level of AST, ALT, ALP, GGT and LDH (Fig. 8B). The free drug combination and liposomal preparations treatments reduced the level of all the indicators to different degrees. In the DEAE-DEX@LSDBC group, all the indicators approached to the normal mice suggesting the more effective protective effects on liver. Besides, oxidative stress as one of the major promoting roles for NAFLD genesis in mice was evaluated by the serum MDA, SOD and GSH levels (Fig. 8C-E). Compared with the Model group, reduced MDA or improved SOD and GSH at liver in the treatment groups, especially in DEAE-DEX@LSDBC, indicating the highly effective ameliorative effects on oxidative stress in mice induced by the high fat and sucrose diet. Collectively, BER and CUR combination in liposomal preparations have been proved to enhance the ameliorative effects on NAFLD in mice, especially in DEAE-DEX@LSDBC.

\section{Protein levels related to oxidative stress and inflammation in liver}

To figure out the mechanisms involved in the ameliorative effects of BER and CUR combination treatments on NAFLD in mice, the level of typical proteins related to 


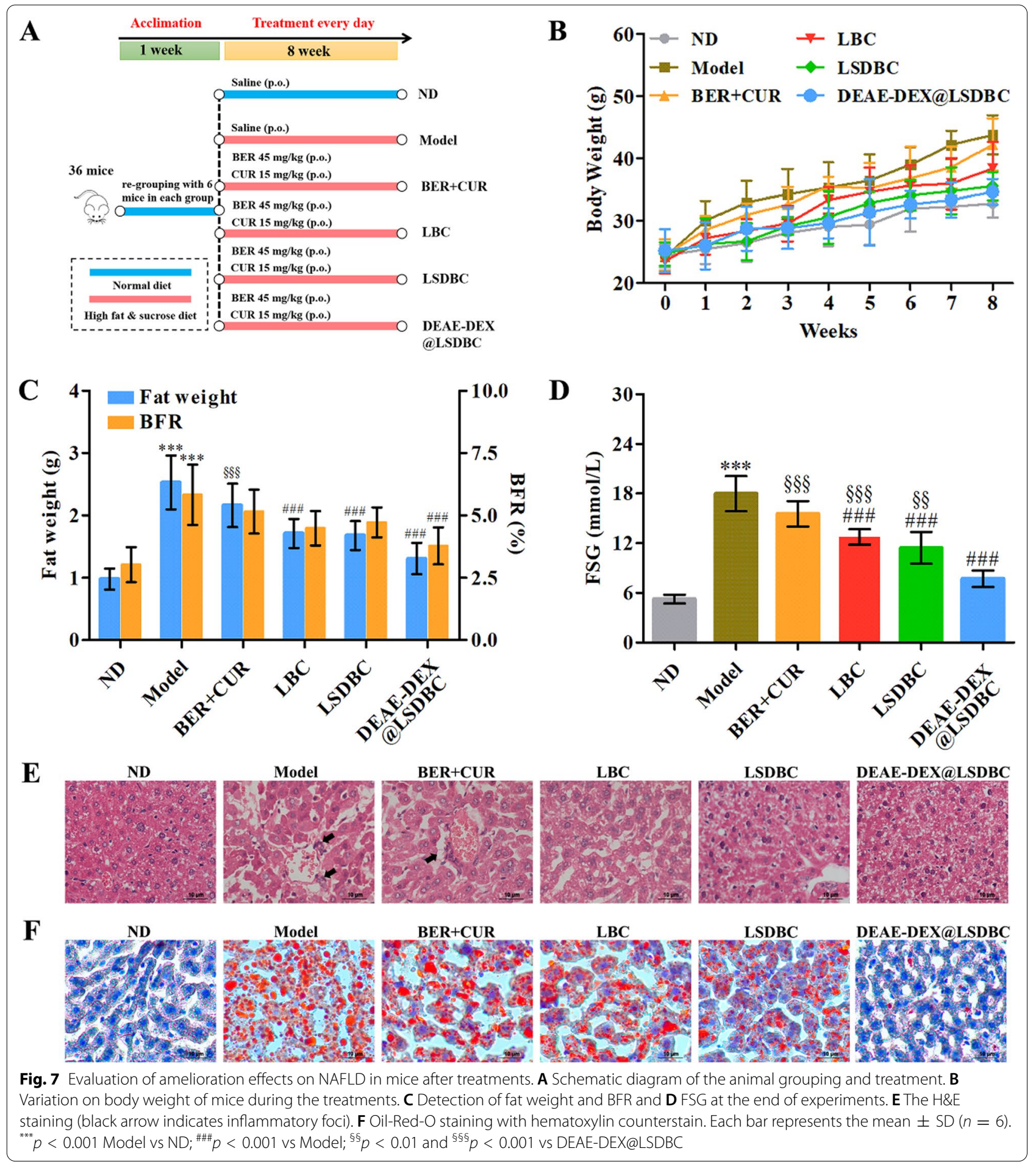

oxidative stress and inflammation were measured, since the anti-oxidative and anti-inflammatory effects are the main mechanisms of BER and CUR. Nuclear factor erythroid-2 related factor 2 (Nrf2) is an important transcription regulator for endogenous antioxidant system.
After drug combination interventions, the translocation of Nrf2 from cytoplasm (Fig. 9A) into nucleus (Fig. 9B) was clearly detected and induced downstream protein expression involving quinone oxidoreductase-1 (NQO1), hemeoxygense-1 (HO-1) and thioredoxin reductase-1 

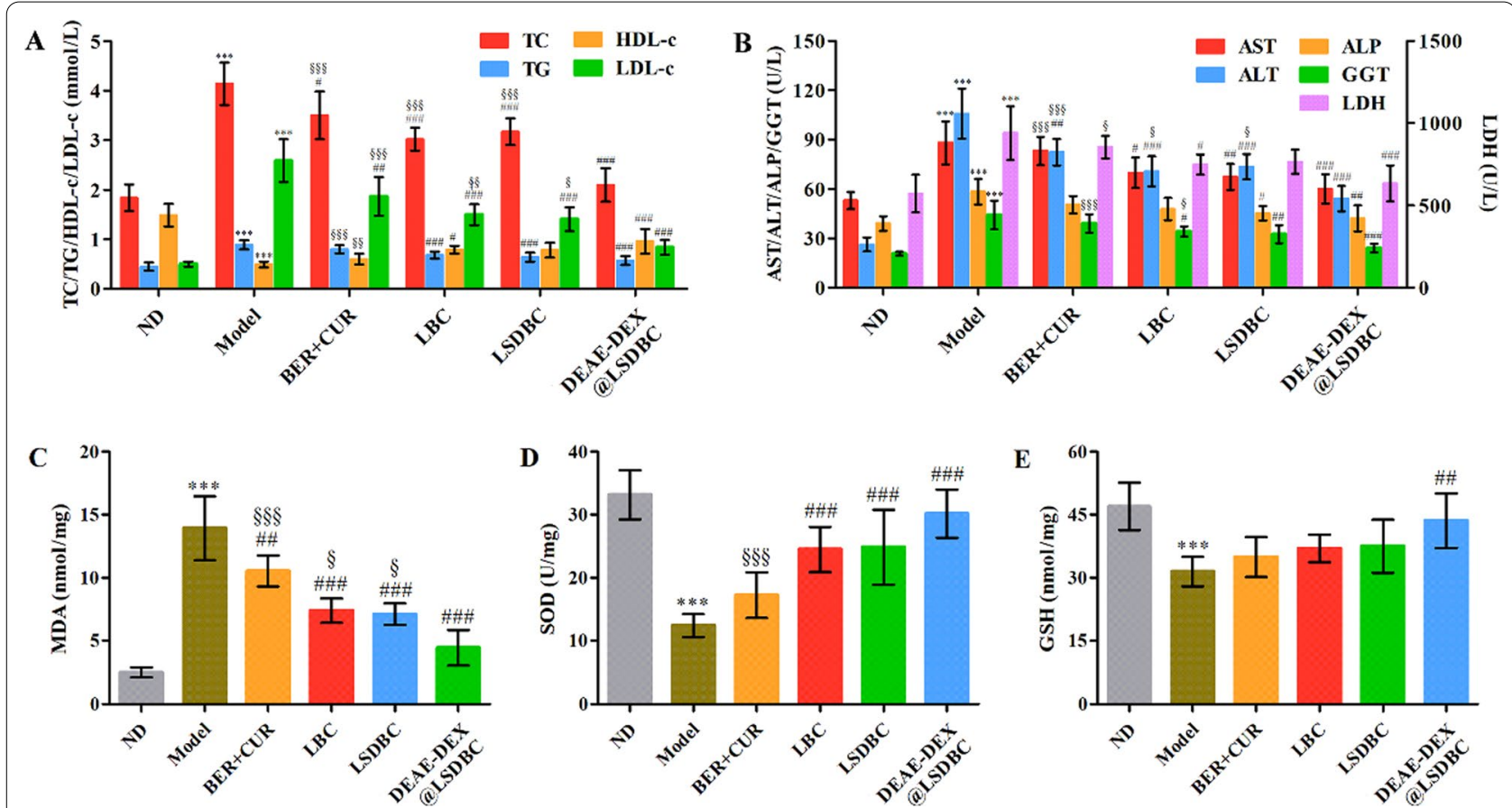

Fig. 8 The biochemical results of different treatment groups. A The serum lipids indicators including TC, TG, HDL-c and LDL-c. B The liver function parameters including AST, ALT, ALP, GGT and LDH. Antioxidant indicators including C MDA, D SOD and $\mathbf{E}$ GSH. Each bar represents the mean \pm SD $(n=6) .{ }^{* * *} p<0.001$ Model vs ND; ${ }^{\#} p<0.05,{ }^{\# \#} p<0.01$ and ${ }^{\# \#} p<0.001$ vs Model; $p<0.05,{ }^{\S \S} p<0.01$, and ${ }^{\S \S \S} p<0.001$ vs DEAE-DEX@LSDBC

(TrxR-1) (Fig. 9C-E). Obviously, DEAE-DEX@LSDBC exerted the most effective promotion effects on the translocation of Nrf2 to nuclear and the upregulation of NQO-1, HO-1 and TrxR-1 among all the groups, which played a potentially antioxidant role against NAFLD.

On the other hand, BER and CUR combination was verified to inhibit the activation of nuclear factor-kappa $B(N F-k B)$ via suppressing the phosphorylation of p65-a subunit of NF- $\kappa B$ (Fig. 9F) and I $\mathrm{IB} \alpha-$ an inhibitor of NF-kB (Fig. 9G). Since phosphorylation of IkB $\alpha$ induces self-degradation that results in phosphorylation of p65 mediated nuclear translocation of NF- $\mathrm{KB}$, the reduced phosphorylation of p 65 and IкB $\alpha$ in the treatment groups inhibited the activation of NF- $\mathrm{kB}$. Meanwhile, enhanced oxidative stress in the Model group trigged upregulation of nucleotide-binding oligomerization domain, leucine-rich repeat or pyrin domain-containing 3 (NLRP3), which activated the inflammasome leading to maturation of Caspase- 1 and IL-1 $\beta$ (Fig. 9H-J). All the drug combination groups exhibited excellent suppression effects on the levels of NLRP3, Caspase-1 and IL-1 $\beta$, especially in
DEAE-DEX@LSDBC group. Thus, DEAE-DEX@LSDBC exerted anti-inflammatory activity on NAFLD in mice via NF- $\mathrm{kB}$ pathway. Moreover, the level of Bax and Bcl-2 were measured to evaluate the apoptosis resulted from oxidative stress and inflammation (Fig. 9K, L). Conspicuously downregulation of Bax and upregulation of Bcl-2 in the treatment groups were also observed in the DEAEDEX@LSDBC group, which were in constant with the trends in the anti-oxidative and anti-inflammatory effects related pathways.

Further analysis of the protein interactions under high fat \& sucrose diet and drug combination intervention was studied via coimmunoprecipitation assay. We carried out immunoprecipitation of thioredoxin-interacting protein (TXNIP) and the blot was revealed with TXNIP, thioredoxin (TRX) and NLRP3 antibodies (Fig. 10A, B). After the drug combination treatment, the formation of TXNIP-TRX complex was promoted and TXNIP-NLRP3 inflammasome was inhibited. Similarly, acetyltransferase p300 was immunoprecipitated and the blot was revealed with p300, p65 and Nrf2 antibodies (Fig. 10C, D). The

(See figure on next page.)

Fig. 9 DEAE-DEX@LSDBC activates the $\mathbf{A}-\mathbf{E}$ Nrf2 signaling, inhibits the $\mathbf{F}-\mathbf{J}$ NLRP3 inflammasome activation and $\mathbf{K}$, $\mathbf{L}$ apoptosis. The indicated protein levels from different groups were detected by western blot and statistical graphs show the relative expression levels of proteins. Each bar represents the mean \pm SD $(n=6) .{ }^{* * *} p<0.001$ Model vs ND; ${ }^{\#} p<0.05,{ }^{\# \#} p<0.01$ and ${ }^{\# \# \#} p<0.001$ vs Model; ${ }^{\S} p<0.05,{ }^{\$ \S} p<0.01$ and ${ }^{\$ \S \S} p<$ 0.001 vs DEAE-DEX@LSDBC 


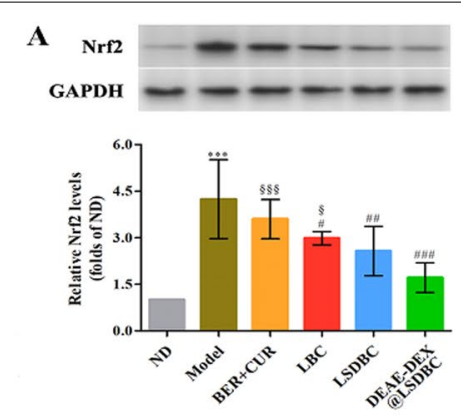

$C_{\text {NQO-1 }}=--\frac{-1}{-}$

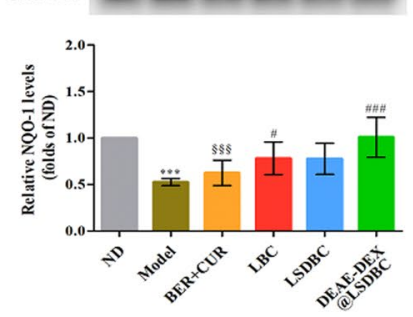

F $\begin{array}{r}\text { phos-p65 } \\ \text { Ser } 536\end{array}$

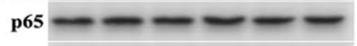

GAPDH -----

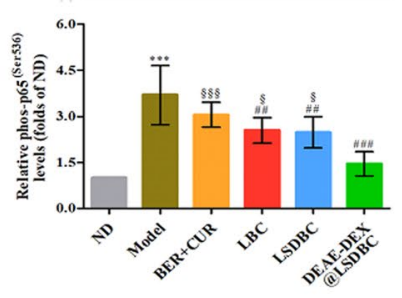

H $_{\text {NLRP3 }}------$

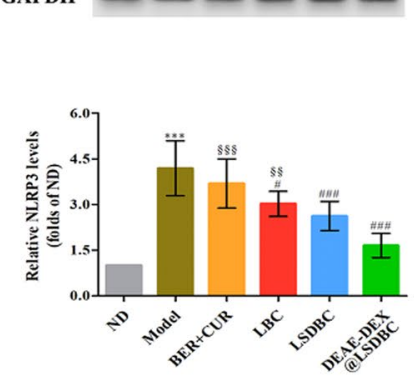

K ${ }_{\text {Bax }}------$
GAPDH $---\cdots$

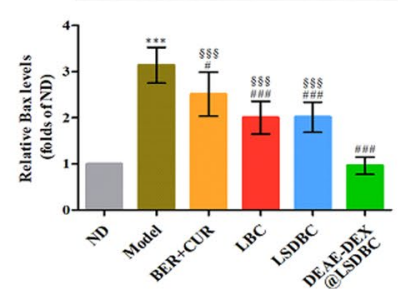

D HO-1 $_{1}$

Caspase-1 p20

GAPDH
B $\quad \mathrm{Nr2}^{-}----$
histone H3 -----

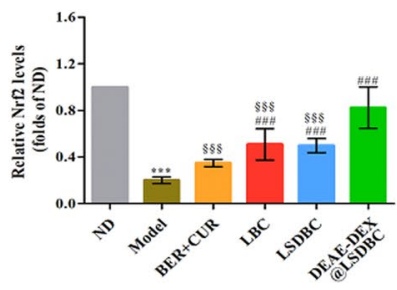

GAPDH $-\cdots--\cdots$

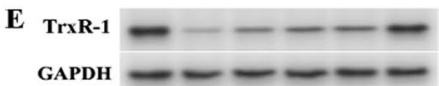
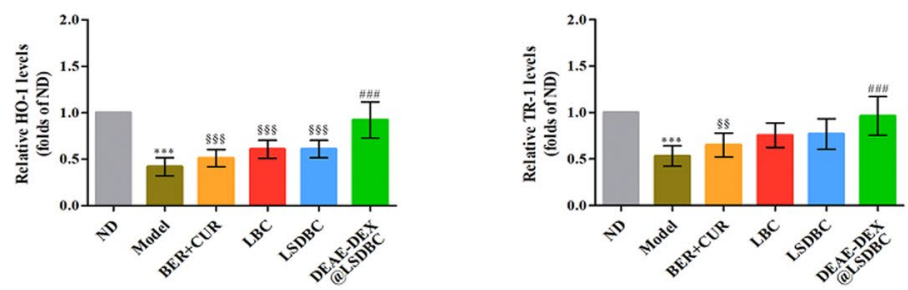

$G$ phos-IrBa
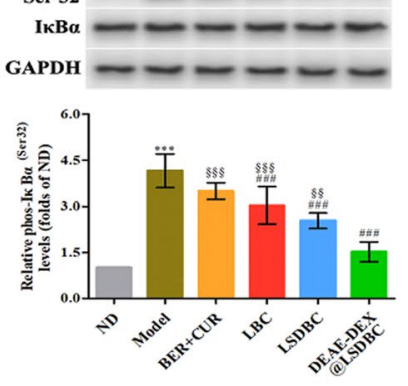

pro-Caspase-1
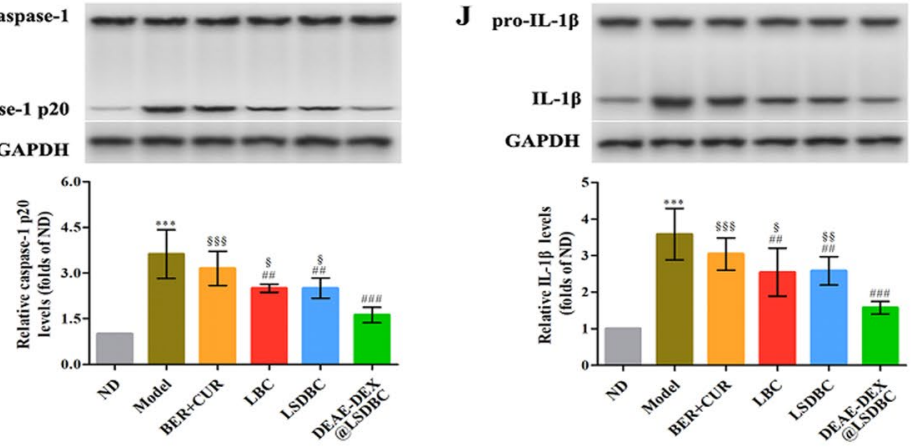

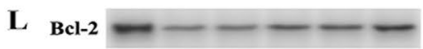

GAPDH $---\div-$

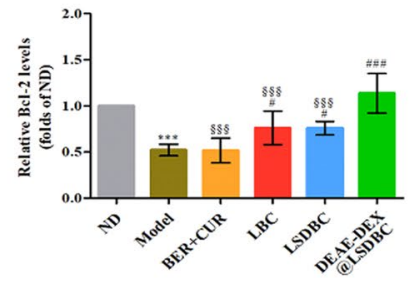

Fig. 9 (See legend on previous page.) 

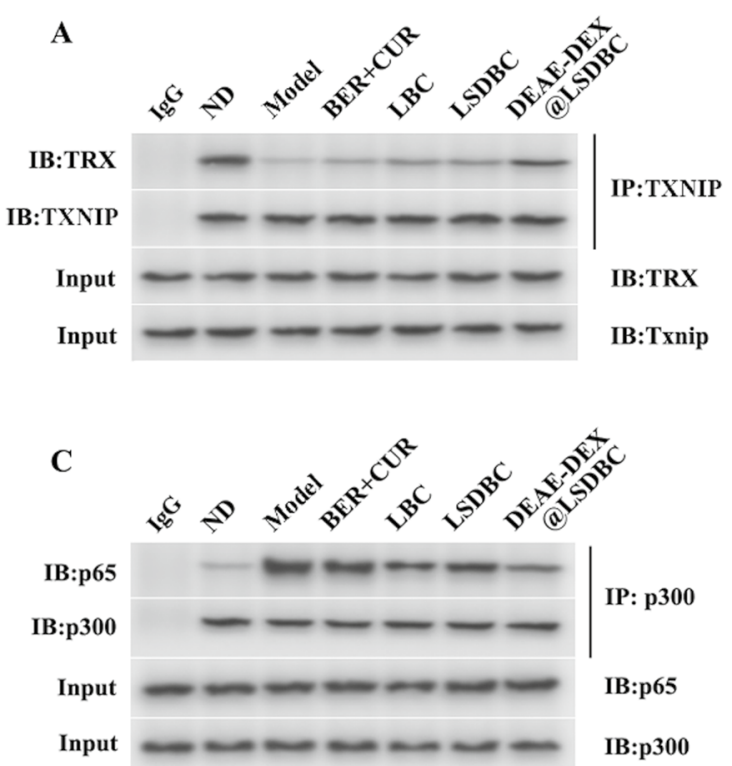

B
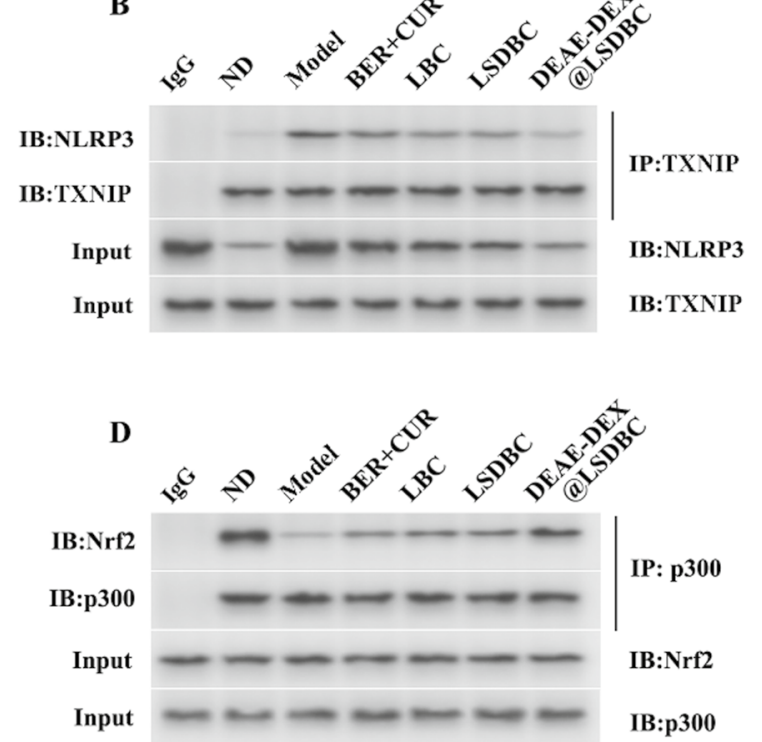

Fig. 10 DEAE-DEX@LSDBC promoted TRX-TXNIP and Nrf2-p300 binding. Liver tissue lysates were analyzed for the interaction of TXNIP and $\mathbf{A}$ TRX or $\mathbf{B}$ NLRP3 and the interaction of p300 and $\mathbf{C} p 65$ or $\mathbf{D}$ Nrf2 by immunoprecipitation

drug combination promoted Nrf2-p300 binding at the expense of p65-p300 interactions, thereby regulating the acetylation of p65 and Nrf2, leading to opposite regulation on the transcriptional activity of p65 and Nrf2. In accordance with the results of protein levels, DEAEDEX@LSDBC showed the most obvious effects on promoting TXNIP-TRX and p300-Nrf2 formation.

Overall, the results suggested that the BER and CUR combination exerted promising ameliorative effects on NAFLD in mice via anti-oxidative and anti-inflammatory activities. It was closely related with the activation of Nrf2 induced antioxidation effects and suppression of NF- $\mathrm{KB}$ induced inflammatory responses. The superior oral absorption, prolonged circulation or synchronized biodistribution of BER and CUR endowed by DEAE-DEX@LSDBC lead to ideal ameliorative effects on NAFLD in mice via enhanced anti-oxidative and antiinflammatory activities.

\section{Conclusions}

A novel DEAE-DEX coated bilosome with BER and CUR combination encapsulated was developed to ameliorate NAFLD in mice. Compared with free BER + CUR, DEAE-DEX@LSDBC improved the stability in the GIT, promoted permeation across mucus and Caco-2 monolayer, enhanced oral absorption and prolonged circulation time of the drug combination. Intriguingly, DEAE-DEX@LSDBC achieved synchronized biodistribution of BER and CUR, especially at liver, which was closely related to the optimal ameliorative effects on
NAFLD that mainly mediated by anti-oxidation and antiinflammation related pathways. Overall, DEAE-DEX@ LSDBC was proved to be an effective preparation for the treatment of NAFLD in mice. The DEAE-DEX coated bilosome provided a promising multilayer nanovesicle for oral administration of free drug combinations.

\section{Methods}

\section{Materials and reagents}

Berberine (BER, purity $\geq 98 \%$ ) and curcumin (CUR, purity $\geq 98 \%$ ) were obtained from Aladdin Reagent Co., Ltd., (Shanghai, China). Soybean lecithin (SPC) was purchased from Lipoid Co., Ltd., (Ludwigshafen am Rhein, Germany). Cholesterol (CHOL) and octadecylamine (ODA) were provided by Sigma-Aldrich Co., Ltd., (St. Louis, Missouri, USA). Sodium deoxycholate (SDC) and diethylaminoethyl dextran (DEAE-DEX) were obtained from Macklin Biochemical Technology Co., Ltd., (Shanghai, China). Detection kits for total cholesterol (TC, A111), triglyceride (TG, A110), high-density lipoprotein cholesterol (HDL-c, A112), low-density lipoprotein cholesterol (LDL-c, A113), aspartate aminotransferase (AST, C001), alanine aminotransferase (ALT, C009), alkaline phosphatase (ALP, A059), $\gamma$-glutamyl transferase (GGT, C017), lactic dehydrogenase (LDH), and glutathione (GSH, A006) were provided by Nanjing Jiancheng Bioengineering Co., Ltd., (Nanjing, China). Detection kits for malondialdehyde (MDA, S013) and superoxide dismutase (SOD, S0103) were obtained from Beyotime Institute of Biotechnology (Shanghai, China). Detection 
kits for Oil Red O staining solution, formaldehyde, alcohol, dimethylbenzene, hematoxylin and eosin (H\&E) stains were provided by Shenggong Bio-engineering Co., Ltd., (Shanghai, China). The Anti-Nrf2 antibody (12,721), Anti-GAPDH antibody (5174), Anti-histone H3 (4499), Anti-HO-1 antibody $(86,806)$, Anti-phosp65(Ser536) antibody (3033), Anti-p65 antibody (8242), Anti-phos-ІкB $\alpha$ (Ser32) antibody (2859), Anti-IкB $\alpha$ antibody (4812), Anti-IL-1 $\beta$ antibody (12,242), Anti-TXNIP antibody $(14,715)$, Anti-p300 antibody $(70,088)$ were provided by Cell Signaling Technology (Boston, USA). The Anti-NQO-1 antibody (ab28947), Anti-TrxR-1 antibody (ab124954), Anti-NLRP3 antibody (ab263899), Anti-Bax antibody (ab32503), Anti-Bcl-2 antibody (ab182858), Goat Anti-Rabbit IgG H\&L (HRP, ab205718), Goat AntiMouse IgG H\&L (HRP, ab205719), Goat Anti-Rabbit IgG H\&L (HRP, ab97051), Anti-Caspase-1 antibody (sc398715) were provided by Santa Cruz Biotechnology Co., Ltd., (Texas, USA). The inorganic chemicals were obtained from J \& K Chemical Co., Ltd., (Beijing, China). Organic solvents of analytical grade were purchased from Sigma-Aldrich Co., Ltd., (St. Louis, Missouri, USA).

\section{Cells and animals}

Caco-2 human colon cancer cell line and LO2 human normal liver cell line were provided by the Cell Bank of the Chinese Academic of Sciences (Shanghai, China). Caco-2 cells and LO2 cells were routinely cultured in DMEM medium (Gibco, California, USA) with $10 \%(\mathrm{v} / \mathrm{v})$ fetal bovine serum (Gibco, Vienna, Austria), $100 \mathrm{U} / \mathrm{mL}$ penicillin and $100 \mathrm{mg} / \mathrm{mL}$ streptomycin. The cells were maintained at $37{ }^{\circ} \mathrm{C}$ in a humidified atmosphere of $95 \%$ air and $5 \% \mathrm{CO}_{2}$.

Male C57BL/6 J mice (20-25 g) and male Kunming mice (20-25 g) were supplied by Guizhou Medical University Laboratory Animal Co., Ltd., [Guiyang, China, Certificate No. SYXK-(Qian) 2018-0001]. The animals were allowed with free access to water and food for 1 -week acclimation. The room temperature was maintained at $23 \pm 2{ }^{\circ} \mathrm{C}$ with $60-70 \%$ humidity with 12 -h light/dark cycle.

\section{Preparation of BER and CUR co-encapsulated bilosomes} The SPC, CHOL, ODA, SDC and CUR in the mass ratio of 20:2:1:3:1 were dissolved in a mixed solvent of chloroform/methanol $(4 / 1, \mathrm{v} / \mathrm{v})$ in around-bottom flask. The solvents were removed dried to form a thin film by a rotary evaporator. Another $2 \mathrm{~h}$ under high vacuum was required to remove the remaining solvent. Because BER was encapsulated by a remote loading method, the $0.3 \mathrm{M}$ $\left(\mathrm{NH}_{4}\right)_{2} \mathrm{SO}_{4}$ solution was added for hydration at $60{ }^{\circ} \mathrm{C}$ for $45 \mathrm{~min}$. After sonication in an ice-water bath at $360 \mathrm{~W}$ for $5 \mathrm{~min}$ ( $3 \mathrm{~s}$ pulse and $3 \mathrm{~s}$ pause) with an ultrasonic cell disruptor (650E, Beidi Institute of Scientific Instruments Co., Ltd., Shanghai, China), the CUR-loaded bilosomes were centrifuged at $1500 \times g$ for $15 \mathrm{~min}$ to remove the free CUR. For the encapsulation of BER, the CURloaded bilosomes were dialysis (molecular weight cut-off, $12 \mathrm{kDa}$ ) against deionized water for $4 \mathrm{~h}$. BER dissolved in deionized water $(7 \mathrm{mg} / \mathrm{mL})$ was mixed with the received CUR-loaded bilosomes at BER/SPC of $7 / 40(\mathrm{mg} / \mathrm{mg})$. The $\mathrm{pH}$ of solution was adjusted by $\mathrm{Na}_{2} \mathrm{CO}_{3}(0.1 \mathrm{M})$ to 7.0 and incubated at $60{ }^{\circ} \mathrm{C}$ for $30 \mathrm{~min}$. Unloaded BER was separated from the bilosomes with both BER and CUR encapsulated (LSDBC) through dialysis against PBS $(0.01 \mathrm{M}$, $\mathrm{pH}$ 7.4) for $4 \mathrm{~h}$. In the optimization of the formulation, the $\mathrm{pH}$ of the external phase in the incubation of BER and the CUR-loaded bilosomes, the incubation time, the mass ratio of SPC/CHOL and SPC/BER were studied in terms of the loading ratio (LR) and loading capability (LC) of both BER and CUR. Besides, the BER and CUR encapsulated liposomes (without SDC inserted) were also prepared as the above optimized procedure and denoted as LBC. After lyophilization, the obtained LBC and LSDBC were stored at $4{ }^{\circ} \mathrm{C}$ until use.

\section{Preparation of DEAE-DEX coated bilosomes}

To attach the positively charged DEAE-DEX on the surface of the LBC bilosomes, the same volume of DEAEDEX in PBS was added dropwise into the bilosomes to different final concentrations $(0.13-0.52 \mathrm{mg} / \mathrm{mL})$ in an ice bath, followed by $100 \mathrm{rpm}$ magnetic stirring for $1 \mathrm{~h}$ at $25{ }^{\circ} \mathrm{C}$. After filtrating with $0.45 \mu \mathrm{m}$ membrane, the BER and CUR encapsulated bilosomes with DEAE-DEX coated (DEAE-DEX@LSDBC) was obtained. The concentration of DEAE-DEX was optimized by evaluating the ability of DEAE-DEX@LSDBC to across the mucus layer. The method was described in the "2.7 Permeation across mucus layer in vitro" item. Lastly, the obtained DEAEDEX@LSDBC were lyophilized and stored at $4{ }^{\circ} \mathrm{C}$ until use.

\section{Characterization of the drug loaded bilosomes}

The LR and LC of BER and CUR were determined by a high-performance liquid chromatography (HPLC) with a UV detector (LC-16, Shimadzu Instruments Co., Ltd., Japan). Briefly, $200 \mu \mathrm{L}$ of the drug loaded bilosomes were added to $9.8 \mathrm{~mL}$ of methanol solution and sonicated for $15 \mathrm{~min}$. After filtering through $0.45 \mu \mathrm{m}$ membrane, the content of CUR loaded was analyzed using a mobile phase of $5 \%$ acetic acid/acetonitrile $(40: 60, \mathrm{v} / \mathrm{v})$ at $\lambda 425 \mathrm{~nm}$. For the detection of BER, a mobile phase of $0.1 \%$ phosphoric acid/acetonitrile $(65: 35, \mathrm{v} / \mathrm{v})$ was used and detected at $\lambda 345 \mathrm{~nm}$. The mobile phase was delivered at $1 \mathrm{~mL} / \mathrm{min}$ through a Hanbon C18 column $(250 \times$ 
$4.6 \mathrm{~mm}$, internal diameter $5 \mu \mathrm{m})$. LR and LC were calculated in accordance with the following equations:

$$
\begin{gathered}
\mathrm{LR}(\text { wt. \% })=\frac{\text { weight of loaded drug }}{\text { weight of feeding drug }} \times 100 \\
\mathrm{LC}(\text { wt. } \%)=\frac{\text { weight of loaded drug }}{\text { theoretical total weight of bilosome }} \times 100
\end{gathered}
$$

The particle size, polydispersity index (PDI) and zeta potential of the prepared preparations were measured by a dynamic laser scattering (90Plus PALS, Brookhaven Instruments Co., Ltd., USA). The Z-average diameter of liposomal preparations was calculated by the automatic mode and the zeta potential was measured more than 10 times run for measurement. The morphology study of LSDBC and DEAE-DEX@LSDBC was implemented with the negative staining method. Film-coated copper grids were used to absorb bilosomes and stained with $1 \%$ phosphotungstic acid solution (pH 7.0). The morphology was observed using a transmission electron microscope (TEM, H-7800, Hitachi Co., Ltd., Japan) at an acceleration voltage of $100 \mathrm{kV}$.

\section{In vitro drug release behavior}

Simulated gastric fluid (SGF) and solutions of intestinal fluid (SIF) were prepared as follows. SGF was prepared by dissolving $16.4 \mathrm{~mL}$ of diluted hydrochloric acid and $10 \mathrm{~g}$ pepsase in $800 \mathrm{~mL}$ of distilled water and then diluted to $1000 \mathrm{~mL}$. For SIF preparation, $6.8 \mathrm{~g}$ monobasic potassium phosphate was firstly dissolved in $500 \mathrm{~mL}$ distilled water and adjusted to $\mathrm{pH} 6.8$ with $0.1 \mathrm{~mol} / \mathrm{L} \mathrm{NaOH}$. Then, SIF was prepared by adding $1 \mathrm{~g}$ trypsin for every $100 \mathrm{~mL}$ of the above solution. Both medium included $0.1 \%$ Tween 80 for solubilization of CUR.

Drug release profile of LBC, LSDBC and DEAE-DEX@ LSDBC was studied with a membrane dialysis technique in SFG at the first $2 \mathrm{~h}$ and then transferred to SIF. Briefly, $1 \mathrm{~mL}$ of redissolved preparations were placed in a dialysis bag (molecular weight cut-off, $12 \mathrm{kDa}$ ) and dispersed in $20 \mathrm{~mL}$ of release medium. In a shaking incubator $\left(100 \mathrm{rpm}, 37^{\circ} \mathrm{C}\right)$, the medium $(0.1 \mathrm{~mL})$ was collected at predetermined time points for $24 \mathrm{~h}$ and the same amount of fresh medium was supplemented. The amount of BER and CUR released in the medium were analyzed by HPLC. All drug release studies meet the requirement of sink condition.

\section{Permeation across mucus layer in vitro}

The permeability of preparations to across the mucus layer were evaluated by the transwell system. Briefly, $0.2 \mathrm{~mL}$ of mucin dissolved in hank's balanced salt solution (HBSS) at $10 \mathrm{mg} / \mathrm{mL}$ was added to the upper chambers of transwell (12 insert/24 well plates, pore size $3 \mu \mathrm{m}$, Millipore, Billerica, MA, USA) and the lower chambers were filled with $0.8 \mathrm{~mL}$ HBSS. Then, $0.2 \mathrm{~mL}$ of BER + CUR and redissolved LBC, LSDBC and DEAE-DEX@LSDBC (at an equivalent concentration of $90 \mu \mathrm{g} / \mathrm{mL}$ BER and $30 \mu \mathrm{g} / \mathrm{mL}$ CUR) were added into the upper chambers, respectively. Incubating in a water bath at $37{ }^{\circ} \mathrm{C}$ and preventing from light, $0.6 \mathrm{~mL}$ solution was collected from the lower chambers for measurements at $0.5,1$ and $2 \mathrm{~h}$, respectively. The concentrations of BER and CUR in the lower chambers were determined by HPLC and the ratio of trans-mucus BER and CUR in different groups were also calculated.

\section{Transcellular permeation assessment on Caco- 2 cell monolayers}

Caco- 2 cells were seeded at a density of $5 \times 10^{4}$ cells/ well in 24-well transwell plates and cultured in DMEM medium with $10 \% \mathrm{v} / \mathrm{v}$ fetal bovine serum for 21 days. The transepithelial electrical resistance (TEER) in Caco-2 cell monolayers was measured by a voltohmmeter (Millicell ERS-2, Millipore Co., Ltd., USA). The TEER values reached to $500 \Omega \mathrm{cm}^{2}$ were used in the following experiment. To prepare samples, BER + CUR, LBC, LSDBC or DEAE-DEX@LSDBC were respectively dissolved in HBSS with $90 \mu \mathrm{g} / \mathrm{mL}$ BER and $30 \mu \mathrm{g} / \mathrm{mL}$ CUR. Then, the DMEM medium were removed and equilibrated with pre-warmed HBSS for $30 \mathrm{~min}$ at $37^{\circ} \mathrm{C}$. The HBSS was replaced with $0.2 \mathrm{~mL}$ samples in the upper chambers and $0.8 \mathrm{~mL}$ fresh HBSS in the lower chambers. After $2 \mathrm{~h}$ incubation, $0.6 \mathrm{~mL}$ solution was withdrawn from the lower chambers and quantitatively analyzed by the HPLC for the content of BER and CUR. The apparent permeability $\left(\mathrm{P}_{\text {app }}\right)$ of BER and CUR were calculated as the following equation:

$$
P_{a p p}=\frac{Q}{A C_{0} t}
$$

where $Q$ was the cumulative amount (ng) of the BER or CUR transported into the lower chambers, $A$ was the Caco-2 cell monolayer area $\left(\mathrm{cm}^{2}\right), C_{0}$ was the BER or CUR concentration $(\mathrm{ng} / \mathrm{mL})$ initially in the upper chambers, and $t$ was the duration time (s) of the permeability experiment.

Besides, different inhibitors were added in the process of permeability experiments to investigate the transcellular mechanism of DEAE-DEX@LSDBC. Briefly, the culture medium was removed and incubated with pre-warmed HBSS for $30 \mathrm{~min}$. Then, the Caco- 2 monolayers were washed and incubated with $4{ }^{\circ} \mathrm{C}$ HBSS or $37{ }^{\circ} \mathrm{C}$ HBSS containing (1) colchicine $(4 \mu \mathrm{g} / \mathrm{mL})$, (2) chlorpromazine $(10 \mu \mathrm{g} / \mathrm{mL})$, (3) genistein $(100 \mu \mathrm{g} / \mathrm{mL})$, (4) cholic acid $(100 \mu \mathrm{M})$ and (5) DEAE-DEX $(0.39 \mathrm{mg} /$ 
$\mathrm{mL}$ ) for $30 \mathrm{~min}$. After that, $0.2 \mathrm{~mL}$ of HBSS containing DEAE-DEX@LSDBC were added in the upper chambers at a final concentration of $90 \mu \mathrm{g} / \mathrm{mL}$ BER and $30 \mu \mathrm{g} / \mathrm{mL}$ CUR and the cell monolayers were incubated at $37^{\circ} \mathrm{C}$ for another $2 \mathrm{~h}$. Then, the concentration of the BER or CUR transported into the lower chambers was measured with a HPLC. The values of $\mathrm{P}_{\text {app }}$ were calculated as described above.

\section{Evaluation on the integrity of Caco-2 cell monolayer}

The impact of preparations on the integrity of the Caco-2 monolayers was measured by the TEER values. After equilibrating in HBSS for $30 \mathrm{~min}$, Caco-2 cell monolayers were measured by an electrical resistance system to determine the TEER values. After that, the HBSS was removed and replaced with $0.2 \mathrm{~mL}$ HBSS containing BER + CUR, LBC, LSDBC or DEAE-DEX@LSDBC at the concentration of $90 \mu \mathrm{g} / \mathrm{mL}$ BER and $30 \mu \mathrm{g} / \mathrm{mL}$ CUR in the upper chambers and $0.6 \mathrm{~mL}$ fresh HBSS in the lower chambers. The TEER values of Caco- 2 monolayers in different groups were measured after 0,1 and $2 \mathrm{~h}$ during the incubation. Subsequently, the upper chambers and lower chambers were washed twice with pre-warmed HBSS. The monolayers were incubated in fresh HBSS again and the final TEER values at $4 \mathrm{~h}$ were measured.

\section{Pharmacokinetics assay}

The male Kunming mice were randomly divided into four groups, and treated with BER + CUR, LBC, LSDBC and DEAE-DEX@LSDBC at dose of $45 \mathrm{mg} / \mathrm{kg}$ BER and $15 \mathrm{mg} / \mathrm{kg}$ CUR via the oral gavage. At specified time points $(0.25,0.5,1,2,4,6,8,12,24 \mathrm{~h})$, mice in each group were anesthetized using inhaled isoflurane with a small animal anesthetic equipment (ZS-MV-IV, Zhongshi Scientific Instruments Co., Ltd., Beijing, China). Then the blood was sampled from the postcava and collected in heparin coated tubes. After centrifugation at $1500 \times g$ for $15 \mathrm{~min}, 200 \mu \mathrm{L}$ plasma from the upper layer was mixed with $800 \mu \mathrm{L}$ methanol and then vortexed $1 \mathrm{~min}$. The mixture was centrifuged at $5000 \times g$ for $10 \mathrm{~min}$ at $4{ }^{\circ} \mathrm{C}$ and the supernatant was analyzed using HPLC. The pharmacokinetic data analysis was performed using the pharmacokinetic software WinNonlin 5.2.1 (Pharsight, Mountain View, CA, USA).

\section{In vivo biodistribution of the preparations}

The study was carried out on the male Kunming mice. For the Kunming mice, BER + CUR, LBC, LSDBC and DEAE-DEX@LSDBC were administered intragastrically at dose of $45 \mathrm{mg} / \mathrm{kg}$ BER and $15 \mathrm{mg} / \mathrm{kg}$ CUR. At specified time points $(1,6,24 \mathrm{~h})$, the mice were anesthetized using small animal anesthetic equipment. The blood was centrifuged to recover the plasma and then analyzed by
HPLC. The heart, liver, spleen, lung and kidney were harvested and weighed. After that, organs were homogenized with PBS in a proportion of $3 \mathrm{~mL}$ PBS per gram of the liver and $6 \mathrm{~mL}$ PBS per gram of the other organs. After centrifugation at $2500 \times g$ for $10 \mathrm{~min}, 100 \mu \mathrm{L}$ supernate of homogenate was mixed with $200 \mu \mathrm{L}$ methanol and vortexed for $1 \mathrm{~min}$. The final solutions were centrifugated at $10,000 \times g$ for $10 \mathrm{~min}$ and the supernatant was analyzed using HPLC.

\section{Animal grouping and treatment}

After acclimation for 1 week, the male C57BL/6 J mice were divided into six groups: one normal diet group (ND group) and five high fat \& sucrose diet groups. The high fat and sucrose diet was made by $40 \%$ fat, $40 \%$ carbohydrate and 20\% protein (D12327, Research Diets, NJ). The ND group was daily treated with saline via the oral gavage for 8 weeks. The five high fat and sucrose diet groups were intragastrically treated with saline (Model group), BER + CUR, LBC, LSDBC and DEAE-DEX@LSDBC for 8 weeks continually. All the formulation groups were treated at dose of $45 \mathrm{mg} / \mathrm{kg}$ BER and $15 \mathrm{mg} / \mathrm{kg}$ CUR. Body weights of mice were recorded every week in the whole course of study.

\section{Preparation of the tissues and serum samples}

After $12 \mathrm{~h}$ fasting, the male C57BL/6 J mice were anaesthetized using small animal anesthetic equipment. The blood was freshly collected from the postcava. Subsequently, the serum was separated by centrifugation at $1000 \times g$ for $10 \mathrm{~min}$ and then stored in a container at $4{ }^{\circ} \mathrm{C}$ for biochemical analysis. To calculate the body fat rate (BFR), the tissue fats and livers of all groups were excised and weighted. One portion of livers were fixed in $10 \%$ phosphate-buffered formalin and the other livers were stored in container at $-80^{\circ} \mathrm{C}$ for further analysis.

\section{Histological assessment}

After immersed in $10 \%$ formalin solution, the harvested livers were embedded in paraffin blocks and sliced for the $H \& E$ staining. The liver sections were stained with OilRed-O and counterstained with hematoxylin to observe the hepatic lipid accumulation.

\section{Biochemical assays}

The fasting serum glucose (FSG) level is the glucose metabolism parameter. Serum lipids indicators includes TC, TG, HDL-c, LDL-c. Liver function parameters includes AST, ALT, ALP, GGT and LDH. Antioxidant indicators includes MDA, SOD and GSH. All the biochemical assays were performed according to the kit supplier's instructions. 


\section{Western blot}

Total protein extracts of liver were obtained by homogenizing and centrifuged at $18,000 \times g$ for $20 \mathrm{~min}$ in $4{ }^{\circ} \mathrm{C}$. The loading amount was calculated depended on the protein concentration which was quantified by a BCA protein assay kit (Beyotime Institute of Biotechnology, Jiangsu, China). Protein lysates were separated by a $10 \%$ sodium dodecyl sulfate-polyacrylamide gel electrophoresis (SDS-PAGE) and transferred to polyvinylidene fluoride (PVDF) membranes (Millipore Co., Ltd., Bedford, MA, USA). Membranes were blocked by $5 \%$ bovine serum albumin (Solarbio, Beijing, China) and incubated overnight with primary antibodies at $4{ }^{\circ} \mathrm{C}$. After that, membranes were washed and incubated with the secondary antibodies. After visualizing with an enhanced chemiluminescence (ECL) kit (7 Sea Biotech, Shanghai, China), digital images of blots were obtained by a GE ImageQuant LAS 500 system (GE Healthcare, Little Chalfont, Buckinghamshire, UK) and analyzed with Image J Software.

\section{Coimmunoprecipitation assay}

Liver tissues were homogenized with a handheld homogenizer with lysis buffer (Beyotime Institute of Biotechnology, P0013) on ice for $30 \mathrm{~min}$ and centrifuged at $12,000 \times g$ at $4{ }^{\circ} \mathrm{C}$ for $15 \mathrm{~min}$. The supernatant fractions were collected and incubated with appropriate antibody $(1 \mu \mathrm{g} / 100 \mu \mathrm{L})$ at $4{ }^{\circ} \mathrm{C}$ overnight and precipitated with protein A/G-agarose beads (Santa Cruz Biotechnology Co., Ltd., sc-2003) for another $4 \mathrm{~h}$ at $4{ }^{\circ} \mathrm{C}$. The beads were washed with the lysis buffer 4 times by centrifugation at $1000 \times g$ at $4{ }^{\circ} \mathrm{C}$. The immunoprecipitated proteins were separated by SDS-PAGE and western blot was performed with the indicated antibodies.

\section{Statistical analysis}

Results were presented in the form of mean \pm standard deviation (SD). For statistical comparison among groups, one-way ANOVA analysis with post hoc Tukey-Kramer test was used with GraphPad Prism software package. The $p$ value less than 0.05 was statistically significant.

\footnotetext{
Abbreviations

ACWC: Animal Care Welfare Committee; ALP: Alkaline phosphatase; ALT: Alanine aminotransferase; AMPK: Adenosine monophosphate activated protein kinase; ASGPR: Asialoglycoprotein receptor; AST: Aspartate aminotransferase; BER: Berberine; BFR: Body fat rate; $\mathrm{CHOL}$ : Cholesterol; CUR : Curcumin; DEAE-DEX: Diethylaminoethyl dextran; DEAE-DEX@LSDBC: DEAE-DEX-coated bilosome with BER and CUR encapsulated; ECL: Enhanced chemiluminescence; FSG: Fasting serum glucose; GGT: $\gamma$-Glutamyl transferase; GIT: Gastrointestinal tract; GSH: Glutathione; HBSS: Hank's balanced salt solution; HCC: Hepatocellular carcinoma; HDL-c: High-density lipoprotein cholesterol; HE: Hematoxylin and eosin; HO-1: Hemeoxygense-1; HPLC: High-performance liquid chromatography; LBC: The BER and CUR encapsulated liposomes without SDC inserted; LC: Loading capability; LDH: Lactic
}

dehydrogenase; LDL-c: Low-density lipoprotein cholesterol; LDLR: Low density lipoprotein receptor; LR: Loading ratio; LSDBC: Bilosomes with both BER and CUR encapsulated; MDA: Malondialdehyde; NAFLD: Non-alcohol fatty liver disease; NASH: Nonalcoholic steatohepatitis; NF-kB: Nuclear factor-kappa B; NLRP3: Pyrin domain-containing 3; NQO-1: Quinone oxidoreductase-1; Nrf2: Nuclear factor erythroid-2 related factor 2; ODA: Octadecylamine; $P_{a p p}$ : Apparent permeability; PDI: Polydispersity index; PVDF: Polyvinylidene fluoride; SDC: Sodium deoxycholate; SDS-PAGE: Sulfate-polyacrylamide gel electrophoresis; SGF: Simulated gastric fluid; SIF: Solutions of intestinal fluid; SOD: Superoxide dismutase; SPC: Soybean lecithin; TC: Total cholesterol; TEER: Transepithelial electrical resistance; TEM: Transmission electron microscope; TG: Triglyceride; TRX: Thioredoxin; TrxR-1: Thioredoxin reductase-1; TXNIP: Thioredoxin-interacting protein.

\section{Supplementary Information}

The online version contains supplementary material available at https://doi. org/10.1186/s12951-021-00979-1.

Additional file 1: Figure S1. Optimization on the LR of LSDBC in terms of (A) external phase $\mathrm{pH}$ and (B) incubation time. The LC of LSDBC was optimized in terms of (C) external phase $\mathrm{pH}$ and (D) incubation time. Each bar represents the mean $\pm \mathrm{SD}(n=3)$. Figure S2. Optimization on the LR of LSDBC in terms of (A) the weight ratio of SPC to $\mathrm{CHOL}$ and (B) SPC to BER. The LC of LSDBC was optimized in terms of $(A)$ the weight ratio of SPC to $\mathrm{CHOL}$ and (B) SPC to BER. Each bar represents the mean $\pm \mathrm{SD}(n=3)$. Figure S3. Transportation of (A) BER and (B) CUR across mucus layer via DEAE-DEX@LSDBC with various amount of DEAE-DEX. Each bar represents the mean $\pm S D(n=3)$. Figure S4. UV spectrum of BER, CUR, LIP, LBC, LSDBC and DEAE-DEX@LSDBC. Figure S5. Cytotoxicity of preparations against Caco-2 cells and LO2 cells. Each bar represents the mean $\pm \mathrm{SD}$ ( $n$ $=3$ ). Figure S6. Synchronized biodistribution of BER and CUR from preparations orally delivery in the male Kunming mice. The T/P ratios for BER and CUR from (A-C) BER+CUR, (D-F) LBC, (G-I) LSDBC and (J-L) DEAEDEX@LSDBC in the heart, liver, spleen, lung and kidney at $(A, D, G, J) 1 \mathrm{~h}$, $(B, E, H, K) 6 \mathrm{~h}$ and $(C, F, I, L) 24$ h after orally administration. Each bar represents the mean $\pm \mathrm{SD}(n=3) .{ }^{*} p<0.05,{ }^{* *} p<0.01$ and ${ }^{* * *} p<0.001$ BER vs CUR in various tissues. Figure S7. Representative morphological images of Oil-Red-O staining on LO2 cells after $12 \mathrm{~h}$ incubation with various preparations (Scar bar $50 \mu \mathrm{m}$ ). Figure S8. Drug release behavior of CUR and BER in both SIF and SGF medium for $24 \mathrm{~h}$. Each symbol represents the mean \pm SD $(n=3)$. Table S1. Pharmacokinetics parameters of BER after oral administration $(n=5)$. Table S2. Pharmacokinetics parameters of CUR after oral administration $(n=5)$.

\section{Acknowledgements}

Not applicable.

\section{Authors' contributions}

YC: investigation, original draft writing, visualization, formal analysis, and data curation. ZJ: investigation and validation. JX: investigation. JZ: visualization and software. RS: investigation and validation. JZ: investigation. YL: investigation. ZG: investigation. JH: investigation. XS: methodology, supervision. QD: investigation, methodology, writing, review and editing, supervision, project administration. JP: conceptualization, methodology, writing and editing, supervision, funding acquisition. All authors read and approved the final manuscript.

\section{Funding}

This work was supported by Excellent Young Talents Plan of Guizhou Medical University (No. 2020-102, China), High-level Overseas Talent Fund for Home Work From Ministry of Human Resources and Social Security (No. RSBLXHGZ202001, China), Distinguished Young Scholars of Nanjing (JQX20008, China) and Foundation for Training Programs of Innovation and Entrepreneurship for Undergraduates of Guizhou Medical University (No. S202010660015, (hina).

Availability of data and materials

All data generated or analyzed during this study are included in this published article and its supplementary information file. 


\section{Declarations}

\section{Ethics approval and consent to participate}

All the animal experiments were conducted according to the National Institutes of Health Guide for the Care and use of Laboratory Animals and were strictly complied with protocols approved by the Animal Care Welfare Committee (ACWC, Guizhou Medical University, No. 2000108). Consent to participate: not applicable.

\section{Consent for publication}

Not applicable.

\section{Competing interests}

The authors declare no competing interests.

\section{Author details}

${ }^{1}$ State Key Laboratory of Functions and Applications of Medicinal Plants, Guizhou Medical University, Guiyang 550014, China. ${ }^{2}$ Key Laboratory of Optimal Utilization of Natural Medicine Resources, School of Pharmaceutical Sciences, Guizhou Medical University, Guiyang 550025, China. ${ }^{3}$ General Clinical Research Center, Nanjing First Hospital, Nanjing Medical University, Nanjing 210006, China. ${ }^{4}$ Department of Clinical Pharmacy, School of Basic Medicine \& Clinical Pharmacy, China Pharmaceutical University, Nanjing 210009, China. ${ }^{5}$ Department of Clinical Laboratory, The First People's Hospital of Guiyang, Guiyang 550002, China. ${ }^{6}$ Nanjing Drum Tower Hospital, the Affiliated Hospital of Nanjing University Medical School, Nanjing 210008, China.

Received: 1 May 2021 Accepted: 27 July 2021

Published online: 04 August 2021

\section{References}

1. Younossi Z, Anstee Q, Marietti M, Hardy T, Henry L, Eslam M, George J, Bugianesi E. Global burden of NAFLD and NASH: trends, predictions, risk factors and prevention. Nat Rev Gastroenterol Hepatol. 2018;15:11-20.

2. Lombardi R, Onali S, Thorburn D, Davidson B, Gurusamy K, Tsochatzis E. Pharmacological interventions for non-alcohol related fatty liver disease (NAFLD): an attempted network meta-analysis. Cochrane Database Syst Rev. 2017:3:CD011640.

3. Younossi Z, Tacke F, Arrese M, Chander Sharma B, Mostafa I, Bugianesi E, Wai-Sun Wong V, Yilmaz Y, George J, Fan J, Vos M. Global perspectives on nonalcoholic fatty liver disease and nonalcoholic steatohepatitis. Hepatology. 2019;69:2672-82.

4. Borrelli A, Bonelli P, Tuccillo FM, Goldfine ID, Evans JL, Buonaguro FM, Mancini A. Role of gut microbiota and oxidative stress in the progression of non-alcoholic fatty liver disease to hepatocarcinoma: current and innovative therapeutic approaches. Redox Biol. 2018;15:467-79.

5. Altamirano-Barrera A, Barranco-Fragoso B, Mendez-Sanchez N. Management strategies for liver fibrosis. Ann Hepatol. 2017:16:48-56.

6. Quesada-Vázquez S, Aragonès G, Del Bas JM, Escoté X. Diet, gut microbiota and non-alcoholic fatty liver disease: three parts of the same axis. Cells. 2020;9:176.

7. Buzzetti E, Pinzani M, Tsochatzis E. The multiple-hit pathogenesis of nonalcoholic fatty liver disease (NAFLD). Metabolism. 2016;65:1038-48.

8. Yan T, Yan N, Wang P, Xia Y, Hao H, Wang G, Gonzalez FJ. Herbal drug discovery for the treatment of nonalcoholic fatty liver disease. Acta Pharm Sin B. 2020;10:3-18.

9. Yao H, Qiao YJ, Zhao YL, Tao XF, Xu LN, Yin LH, Qi Y, Peng JY. Herbal medicines and nonalcoholic fatty liver disease. World J Gastroenterol. 2016:22:6890-905.

10. Xu X, Yi H, Wu J, Kuang T, Zhang J, Li Q, Du H, Xu T, Jiang G, Fan G. Therapeutic effect of berberine on metabolic diseases: both pharmacological data and clinical evidence. Biomed Pharmacother. 2021;133:110984

11. Xing L, Zhang L, Liu T, Hua Y, Zheng P, Ji G. Berberine reducing insulin resistance by up-regulating IRS-2 mRNA expression in nonalcoholic fatty liver disease (NAFLD) rat liver. Eur J Pharmacol. 2011;668:467-71.
12. Yuan X, Wang J, Tang X, Li Y, Xia P, Gao X. Berberine ameliorates nonalcoholic fatty liver disease by a global modulation of hepatic mRNA and IncRNA expression profiles. J Transl Med. 2015;13:24-35.

13. Mai W, Xu Y, Xu J, Zhao D, Ye L, Yu G, Wang Z, Lu Q, Lin J, Yang T, et al. Berberine inhibits nod-like receptor family pyrin domain containing 3 inflammasome activation and pyroptosis in nonalcoholic steatohepatitis via the ROS/TXNIP axis. Front Pharmacol. 2020;11:185-200.

14. Zhu X, Bian H, Gao X. The potential mechanisms of berberine in the treatment of nonalcoholic fatty liver disease. Molecules. 2016:21:1335-48.

15. Zabihi N, Pirro M, Johnston T, Sahebkar A. Is there a role for curcumin supplementation in the treatment of non-alcoholic fatty liver disease? The data suggest yes. Curr Pharm Des. 2017;23:969-82.

16. Inzaugarat ME, De Matteo E, Baz P, Lucero D, Garcia CC, Gonzalez Ballerga E, Daruich J, Sorda JA, Wald MR, Chernavsky AC. New evidence for the therapeutic potential of curcumin to treat nonalcoholic fatty liver disease in humans. PLoS ONE. 2017;12:e0172900.

17. Feng WW, Kuang SY, Tu C, Ma ZJ, Pang JY, Wang YH, Zang QC, Liu TS, Zhao $Y L$, Xiao XH, Wang JB. Natural products berberine and curcumin exhibited better ameliorative effects on rats with non-alcohol fatty liver disease than lovastatin. Biomed Pharmacother. 2018:99:325-33.

18. Rad SZK, Rameshrad M, Hosseinzadeh H. Toxicology effects of berberis vulgaris (barberry) and its active constituent, berberine: a review. Iran J Basic Med Sci. 2017;20:516-29.

19. Kim Y, Clifton P. Curcumin, cardiometabolic health and dementia. Int J Environ Res Public Health. 2018;15:2093-127.

20. Nelson KM, Dahlin JL, Bisson J, Graham J, Pauli GF, Walters MA. The essential medicinal chemistry of curcumin. J Med Chem. 2017:60:1620-37.

21. Cui HX, Hu YN, Li JW, Yuan K, Guo Y. Preparation and evaluation of antidiabetic agents of berberine organic acid salts for enhancing the bioavailability. Molecules. 2018;24:103-12.

22. Dawidczyk C, Kim C, Park J, Russell L, Lee K, Pomper M, Searson P. Stateof-the-art in design rules for drug delivery platforms: lessons learned from FDA-approved nanomedicines. J Control Release. 2014;187:133-44.

23. Tang W, Tang W, Szeitz A, Kulkarni J, Cullis P, Li S. Systemic study of solvent-assisted active loading of gambogic acid into liposomes and its formulation optimization for improved delivery. Biomaterials. 2018;166:13-26.

24. Elnaggar $Y$, Omran $S$, Hazzah $H$, Abdallah O. Anionic versus cationic bilosomes as oral nanocarriers for enhanced delivery of the hydrophilic drug risedronate. Int J Pharm. 2019;564:410-25.

25. Li D, Zhuang J, Yang Y, Wang D, Yang J, He H, Fan W, Banerjee A, Lu Y, Wu $W$, et al. Loss of integrity of doxorubicin liposomes during transcellular transportation evidenced by fluorescence resonance energy transfer effect. Colloids Surf B Biointerfaces. 2018;171:224-32.

26. Hu S, Niu M, Hu F, Lu Y, Qi J, Yin Z, Wu W. Integrity and stability of oral liposomes containing bile salts studied in simulated and ex vivo gastrointestinal media. Int J Pharm. 2013;441:693-700.

27. Aburahma M. Bile salts-containing vesicles: promising pharmaceutical carriers for oral delivery of poorly water-soluble drugs and peptide/ protein-based therapeutics or vaccines. Drug Deliv. 2016;23:1847-67.

28. Niu M, Tan Y, Guan P, Hovgaard L, Lu Y, Qi J, Lian R, Li X, Wu W. Enhanced oral absorption of insulin-loaded liposomes containing bile salts: a mechanistic study Int J Pharm. 2014:460:119-30.

29. D'Souza A, Devarajan P. Asialoglycoprotein receptor mediated hepatocyte targeting — strategies and applications. J Control Release 2015;203:126-39.

30. Hashida M, Nishikawa M, Yamashita F, Takakura Y. Cell-specific delivery of genes with glycosylated carriers. Adv Drug Deliv Rev. 2001;52:187-96.

31. Chen D, Xia D, Li X, Zhu Q, Yu H, Zhu C, Gan Y. Comparative study of Pluronic $\left({ }^{\circledR}\right)$ F127-modified liposomes and chitosan-modified liposomes for mucus penetration and oral absorption of cyclosporine A in rats. Int J Pharm. 2013:449:1-9.

\section{Publisher's Note}

Springer Nature remains neutral with regard to jurisdictional claims in published maps and institutional affiliations. 